\title{
Higgs inflation and the cosmological constant
}

\author{
Fred Jegerlehner,
}

\author{
Humboldt-Universität zu Berlin, Institut für Physik, \\ Newtonstrasse 15, D-12489 Berlin, Germany \\ Deutsches Elektronen-Synchrotron (DESY), \\ Platanenallee 6, D-15738 Zeuthen, Germany
}

\begin{abstract}
We discuss the impact of the Higgs discovery and its revealing a very peculiar value for the Higgs mass. It turns out that the Higgs not only induces the masses of all SM particles, the Higgs, given its special mass value, is the natural candidate for the inflaton and in fact is ruling the evolution of the early universe, by providing the necessary dark energy which remains the dominant energy density. In a previous paper I have shown that running couplings not only allow us to extrapolate SM physics up to the Planck scale, but equally important they are triggering the Higgs mechanism when the universe cools down to lower temperatures. This is possible by the fact that the bare mass term in the Higgs potential changes sign at about $\mu_{0} \simeq 1.4 \times 10^{16} \mathrm{GeV}$ and in the symmetric phase is enhanced by quadratic terms in the Planck mass. Such a huge Higgs mass term is able to play a key role in triggering inflation in the early universe. In this article we extend our previous investigation by working out the details of a Higgs inflation scenario. We show how different terms contributing to the Higgs Lagrangian are affecting inflation. Given the SM and its extrapolation to scales $\mu>\mu_{0}$ we find a calculable cosmological constant $V(0)$ which is weakly scale dependent and actually remains large during inflation. This is different to the Higgs fluctuation field dependent $\Delta V(\phi)$, which decays exponentially during inflation, and actually would not provide a sufficient amount of inflation to solve the CMB horizon problem. The fluctuation field has a different effective mass which shifts the bare Higgs transition point to a lower value $\mu_{0}^{\prime} \simeq 7.7 \times 10^{14} \mathrm{GeV}$. We also show that for SM inflation standard slow-roll inflation criteria are obsolete. Miraculously, the huge difference between bare and renormalized cosmological constant is nullified either by the running of the SM couplings or by vacuum rearrangement somewhat before the Higgs phase transition takes place. This solves the notorious cosmological constant problem. Like in the case of the standard hierarchy problem concerning the quadratic divergences, also the quartically divergent vacuum energy exhibits a coefficient function which exhibits a zero very close to the zero of the quadratic coefficient function. While the Higgs today is only talking very weakly to the rest of the world, in the early universe it was all-dominating and shaping the universe to look as we see it today. The role of the Higgs in reheating and baryogenesis is emphasized. SM inflation implies reheating by production of top-anti-top pairs predominantly.
\end{abstract}


In our scenario inflation and the EW phase transition happen not far below the Planck scale where baryon and lepton number violating dimension 6 operators, suppressed by at most $\left(\mu / M_{\mathrm{Pl}}\right)^{2}>\left(\mu_{0} / M_{\mathrm{Pl}}\right)^{2}=10^{-6}$, provide sufficient $B$ and $L$ violation to trigger baryogenesis. The heavy "charged" Higgs decays $H^{+} \rightarrow t \bar{d}, u \bar{b}$ and $H^{-} \rightarrow d \bar{t}, b \bar{u}$, proportional to the CP-violating CKM elements $V_{t d}$ and $V_{b u}$ or their conjugates, together with the fact that the Higgs transition and its closely following EW phase transition is driving the system out of equilibrium, provide the proper condition for baryogenesis to derive from SM physics. After the EW phase transition the now heavy flavors decay into the lighter ones ending up as normal matter.

PACS numbers:14.80.Bn, 11.10.Gh, 98.80.Cq, 98.80.Es, 11.30.Fs

Keywords: Higgs mechanism; inflation; cosmological constant; baryogenesis

\section{Introduction}

The discovery of the Higgs by ATLAS [1] and CMS [2] at the LHC, together with the fact that new physics still did not show up, already has changed the paradigm about the path to physics at the high energy scale. The Standard Model (SM) of particle physics [3, 4, appears to be finalized by the Higgs sector [5], as it has been required by the theory of mass generation, and all the main SM parameters are known rather accurately by now [6]. In Ref. [7] we have studied the impact of this new situation:

1) The present status of the SM strengthens the status of the SM as a low energy effective (LEESM) theory of a cutoff system residing at the Planck scale, with the Planck mass $M_{\mathrm{Pl}} \simeq 1.22 \times 10^{19} \mathrm{GeV}$ as a cutoff. It renders the now finite relationship between bare and renormalized parameters to have a precise physical meaning. We thus can calculate the parameters of the bare system residing at the Planck scale.

2) The SM very likely, within present input parameter uncertainties, remains a self-consistent QFT in the perturbative regime up to very close to the Planck scale, with a stable Higgs vacuum.

3) The quadratically enhanced Higgs potential mass counterterm has a known scale dependent coefficient, which changes sign at about $\mu_{0} \simeq 1.4 \times$ $10^{16} \mathrm{GeV}$. The sign-flip is triggering the Higgs mechanism, which means that the SM in the early universe has been in the symmetric phase with four physical very heavy Higgses, while all other SM particles are essentially massless. At the Higgs transition point the difference between bare and renormalized masses is nullified, such that the bare short distance world before the phase transition matches the renormalized low energy world after the phase transition.

4) Before the universe has cooled down to undergo the Higgs phase transition, the Higgs is triggering inflation and provides the necessary large darkenergy term corresponding to a large bare cosmological constant. 
In Ref. 8 we emphasized that the SM in the broken phase has no hierarchy problem. In contrast, in the unbroken phase (in the early universe) the quadratic enhancement of the mass term in the Higgs potential is what promotes the Higgs to be the inflaton scalar field. Thus the "quadratic divergences" provide the necessary condition for the explanation of the inflation profile as extracted from Cosmic Microwave Background (CMB) data 9 .

Note that in the unbroken phase, which exists from the Planck scale down to the Higgs transition not very far below the Planck scale, the bare theory is the physical one and a hierarchy or fine-tuning problem is not an issue there.

Standard Model Higgs vacuum stability bounds have been studied some time ago in Ref. [10,11, for example. Surprisingly, the Higgs mass determined by the LHC experiments revealed a value which just matched or very closely matched expectations from vacuum stability bounds. This has then been elaborated in a number of papers [12 23. While a majority of (not independent) analyses just find sharply missing vacuum stability, which means that new physics must be there to remedy the unstable situation, some analyses obtain results compatible with vacuum stability up to the Planck scale [7,23, 24]. The main issue is the precise value of the top-quark Yukawa coupling, which we find slightly smaller than some other analyses. Differences may be related to issues concerning the mass definition of the top-quark [25] and the proper evaluation of the on-shell versus $\overline{\mathrm{MS}}$ matching conditions as analyzed e.g. in Ref. 24 or more recently in Ref. 22]. Our study is based on input values listed in Table 1, which also lists corresponding input values obtained in Ref. 22. Stable vacuum solutions have been found independently in Ref. 23].

Table 1: $\overline{\mathrm{MS}}$ parameters at various scales for $M_{H}=126 \mathrm{GeV}$ and $\mu_{0} \simeq$ $1.4 \times 10^{16} \mathrm{GeV} . C_{1}$ and $C_{2}$ are the one- and two-loop coefficients of the quadratic divergence Eqs. (2) and (3), respectively. The last two columns show corresponding results from Ref. [22].

\begin{tabular}{ccccc||cr}
\hline \hline coupling $\backslash$ scale & $M_{Z}$ & $M_{t}$ & $\mu_{0}$ & $M_{\mathrm{Pl}}$ & $M_{t}[22]$ & $M_{\mathrm{Pl}}[22]$ \\
\hline$g_{3}$ & 1.2200 & 1.1644 & 0.5271 & 0.4886 & 1.1644 & 0.4873 \\
$g_{2}$ & 0.6530 & 0.6496 & 0.5249 & 0.5068 & 0.6483 & 0.5057 \\
$g_{1}$ & 0.3497 & 0.3509 & 0.4333 & 0.4589 & 0.3587 & 0.4777 \\
$y_{t}$ & 0.9347 & 0.9002 & 0.3872 & 0.3510 & 0.9399 & 0.3823 \\
$y_{b}$ & 0.0238 & 0.0227 & 0.0082 & 0.0074 & & \\
$y_{\tau}$ & 0.0104 & 0.0104 & 0.0097 & 0.0094 & & \\
$\sqrt{\lambda}$ & 0.8983 & 0.8586 & 0.3732 & 0.3749 & 0.8733 & i 0.1131 \\
$\lambda$ & 0.8070 & 0.7373 & 0.1393 & 0.1405 & 0.7626 & -0.0128 \\
$C_{1}$ & -6.768 & -6.110 & 0 & 0.2741 & & \\
$C_{2}$ & -6.672 & -6.217 & 0 & 0.2845 & & \\
$m[\mathrm{GeV}]$ & 89.096 & 89.889 & 97.278 & 96.498 & 97.278 & \\
\hline
\end{tabular}

In a LEESM scenario the only quadratic divergences show up in the renor- 
malization of the mass term in the Higgs potential

$$
m_{0}^{2}=m^{2}+\delta m^{2} ; \delta m^{2}=\frac{\Lambda^{2}}{32 \pi^{2}} C
$$

which communicates the relationship between the bare $m_{0}$ and the renormalized mass $m$. The one-loop coefficient function [26] $C_{1}$ may be written as

$$
C_{1}=2 \lambda+\frac{3}{2} g^{\prime 2}+\frac{9}{2} g^{2}-12 y_{t}^{2},
$$

and is uniquely determined by dimensionless couplings. Surprisingly, taking into account the running of the SM couplings, which are not affected by quadratic divergences such that standard RG equations apply, the coefficient of the quadratic divergence of the Higgs mass counterterm exhibits a zero. This has been emphasized in Ref. [27]. The next-order correction, first calculated in Refs. [28, 29],

$$
\begin{aligned}
C_{2}= & C_{1}+\frac{\ln \left(2^{6} / 3^{3}\right)}{16 \pi^{2}}\left[18 y_{t}^{4}+y_{t}^{2}\left(-\frac{7}{6} g^{\prime 2}+\frac{9}{2} g^{2}-32 g_{s}^{2}\right)\right. \\
& \left.-\frac{87}{8} g^{\prime 4}-\frac{63}{8} g^{4}-\frac{15}{4} g^{2} g^{\prime 2}+\lambda\left(-6 y_{t}^{2}+g^{\prime 2}+3 g^{2}\right)-\frac{2}{3} \lambda^{2}\right],
\end{aligned}
$$

numerically does not change significantly the one-loop result. For $M_{H}=126 \mathrm{GeV}$, and given our set of $\overline{\mathrm{MS}}$ input parameters at the scale $M_{Z}$, the zero of $C_{1}$ is at $\mu_{0} \simeq 1.4 \times 10^{16} \mathrm{GeV}$ the one of $C_{2}$ at $\mu_{0} \simeq 1.8 \times 10^{16} \mathrm{GeV}$. For the same Higgs mass the RG $\beta$-function $\beta_{\lambda}$ has a zero at $1.3 \times 10^{17} \mathrm{GeV}$. Since the difference between $C_{1}$ and $C_{2}$ is small, we will adopt $C_{1}$ and the corresponding value for $\mu_{0}$, in what follows. For later use we also list the zeros for $C_{i}^{\prime}=C_{i}+\lambda$ and $X_{i}=\frac{1}{8}\left(2 C_{i}^{\prime}-\lambda\right)$ (see Eqs.(39) and (63) below) in Table 2 .

Table 2: The location of the zeros of $C_{i}, C_{i}^{\prime}=C_{i}+\lambda$ and $X_{i}=\frac{1}{8}\left(2 C_{i}^{\prime}-\lambda\right)$ as a function of scale in $\mathrm{GeV}$, at one $(i=1)$ and two $(i=2)$ loops. Parameters as in Table 1 .

\begin{tabular}{cc|cc|cc}
\hline \hline$C_{1}$ & $C_{2}$ & $C_{1}^{\prime}$ & $C_{2}^{\prime}$ & $X_{1}$ & $X_{2}$ \\
\hline $1.42 \times 10^{16}$ & $1.82 \times 10^{16}$ & $7.77 \times 10^{14}$ & $9.94 \times 10^{14}$ & $3.25 \times 10^{15}$ & $4.15 \times 10^{15}$ \\
\hline
\end{tabular}

Above the transition point the number of massless degrees of freedom (radiation) of the SM consists of $g_{f}=90$ fermionic degrees of freedom and $g_{b}=24$ bosonic ones such that the effective number of degrees of freedom is given by

$$
g_{*}(T)=g_{b}(T)+\frac{7}{8} g_{f}(T)=102.75,
$$

the factor $7 / 8$ accounts for the Pauli exclusion principle which applies for the fermions. The four Higgses in the symmetric phase have equal masses, and are very heavy. Highly relativistic particles contribute to the radiation density

$$
\rho_{\mathrm{rad}}(T)=\frac{\pi^{2}}{30} g_{*}(T) T^{4}
$$



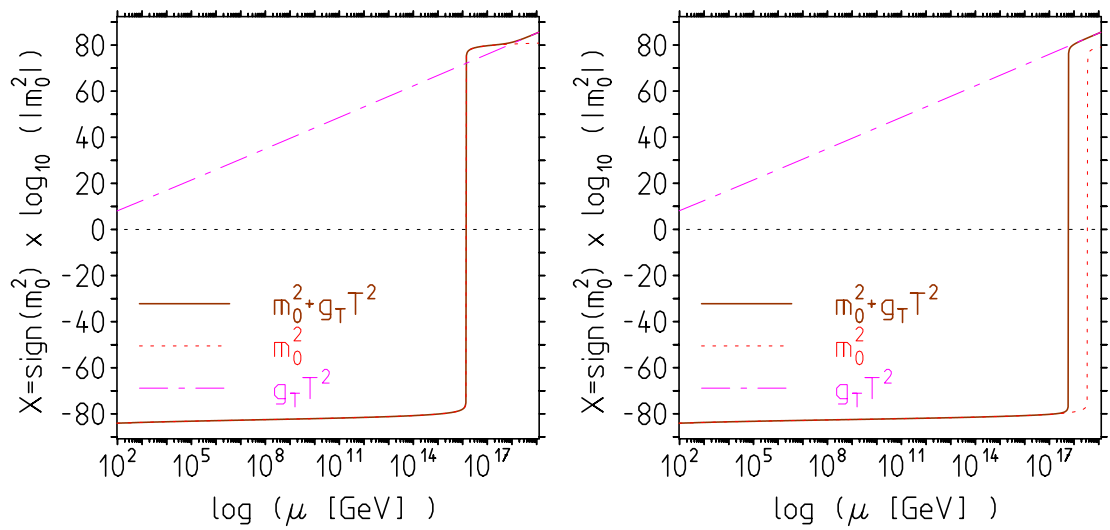

Figure 1: The role of the effective bare mass in the finite temperature SM. Left: for $\mu_{0} \sim 1.4 \times 10^{16} \mathrm{GeV}\left(M_{H} \sim 126 \mathrm{GeV}, M_{t} \sim 173.5 \mathrm{GeV}\right)$. Right: finite temperature delayed transition for $\mu_{0} \sim 6 \times 10^{17} \mathrm{GeV}\left(M_{H} \sim 124 \mathrm{GeV}\right.$, $\left.M_{t} \sim 175 \mathrm{GeV}\right)$, the $m_{0}^{2}$ term alone is flipping at about $\mu_{0} \sim 3.5 \times 10^{18} \mathrm{GeV}$. Inflation requires $m_{0}^{2}>g_{T} T^{2}$ !

according to the Stefan-Boltzmann law.

In the early hot phase of the universe finite temperature effects play an important role. They in particular affect the electroweak (EW) phase transition as well as inflation 30 33. The leading effects enter the finite temperature potential

$$
V(\phi, T)=\frac{1}{2}\left(g_{T} T^{2}-\mu_{\phi}^{2}\right) \phi^{2}+\frac{\lambda}{24} \phi^{4}+\cdots .
$$

by a modified effective mass term. In the $\mathrm{SM} g_{T}$ is given by [33]

$$
g_{T}=\frac{1}{16}\left[3 g^{2}+g^{\prime 2}+4 y_{t}^{2}+\frac{2}{3} \lambda\right]
$$

and with the couplings given in Table 1 we estimate $g_{T} \approx 0.0980$ at $M_{\mathrm{Pl}}$. RG methods allow us to calculate the mass parameter $\mu_{\phi}^{2}$, which in the symmetric phase, at scales $\mu>\mu_{0}$, is given by the symmetric bare mass term

$$
-\mu_{\phi}^{2} \rightarrow m_{0}^{2}=m^{2}+\delta m^{2}>0 .
$$

The EW phase transition, requiring $m_{\text {eff }}^{2}=g_{T} T^{2}+m_{0}^{2}<0$ can only take place after the Higgs transition $m_{0}^{2}>0 \rightarrow m_{0}^{2}<0$, whereby it is important that $m_{0}^{2}$ is large due to the quadratic enhancement of the mass terms. This implies that the Higgs transition essentially triggers the EW phase transition to happen at a very high scale not far below $\mu_{0}$, as we illustrate in Fig. 1. What we call Higgs transition corresponds to the would-be EW phase transition in the zero temperature SM. Standard EW phase transition scenarios (see e.g. Refs. [33-37] 
and references therein) assume $\mu_{\phi}^{2}>0$ in the finite temperature potential (6) to hold up to the Planck scale.

It should be noticed here that in the symmetric phase we actually do not know the parameter $m^{2}$ which corresponds to the renormalized mass. The only possibility to constrain it phenomenologically is via information we have on inflation. We assume it to be small relative to the quadratically enhanced $\delta m^{2}$. Information extracted from CMB fluctuations on the inflaton mass yielded estimates like

$$
m_{\phi} \sim 10^{-6} M_{\mathrm{Pl}},
$$

which essentially supports our assumption.

It is well known that, in principle, the Higgs could be the scalar inflaton field, which is able to explain the phenomenon of inflation in the early universe 38 44] (see also the recent Refs. 45,46] and references therein). Inflation requires an exponential growth $a(t) \propto \mathrm{e}^{H t}$ of the Friedman-Robertson-Walker (FRW) radius $a(t)$ of the universe, where $H(t)=\dot{a} / a(t)$ is the Hubble constant at cosmic time $t$. Data strongly supporting the existence of an inflation era in the evolution of the universe are provided by CMB observations, most recently from the Planck mission (see Ref. [9] and references therein). The SM Higgs affects the evolution of the universe by its contribution to the energy-momentum tensor. Given the Higgs Lagrangian

$$
\mathcal{L}(\phi)=\frac{1}{2} g^{\mu \nu} \partial_{\mu} \phi \partial_{\nu} \phi-V(\phi),
$$

its contribution to the symmetric energy-momentum tensor

$$
\Theta^{\mu}{ }_{\nu}=\frac{\partial \mathcal{L}}{\partial\left(\partial_{\mu} \phi\right)} \partial_{\nu} \phi-\delta^{\mu}{ }_{\nu} \mathcal{L}
$$

reads

$$
\Theta_{\mu \nu}=\frac{1}{2} \partial_{\mu} \phi \partial_{\nu} \phi+\frac{1}{2}\left(g^{\rho \sigma} \partial_{\rho} \phi \partial_{\sigma} \phi\right) g_{\mu \nu}+V(\phi) g_{\mu \nu} .
$$

In the ground state the gradient terms of $\phi$ do not contribute and we obtain

$$
T_{\mu \nu}^{\mathrm{vac}} \equiv\left\langle 0\left|\Theta_{\mu \nu}\right| 0\right\rangle=\rho_{\mathrm{vac}} g_{\mu \nu}=V\left(\phi_{0}\right) g_{\mu \nu} .
$$

Actually, in the ground state this is the only covariant and covariantly conserved form possible. Here, by the Einstein equation

$$
R_{\mu \nu}-\frac{1}{2} R g_{\mu \nu}=\kappa T_{\mu \nu},
$$

the Higgs directly talks to gravity! This is true in any case in the symmetric phase of the SM where1

$$
T_{\mu \nu \mathrm{SM}}^{\mathrm{vac}}=-\left\langle 0\left|\mathcal{L}_{\mathrm{SM}}\right| 0\right\rangle g_{\mu \nu}=V\left(\phi_{0}\right) g_{\mu \nu} .
$$

\footnotetext{
${ }^{1}$ At later stages, when long range phenomema come into play and the SM undergoes spontaneous symmery breaking, other vacuum condensates participate, besides the Higgs VEV $\langle H\rangle=v$ generated at the EW phase transiton, in particular the quark and gluon condensates after the QCD phase transition.
} 
Indeed the Planck medium factually is unifying gravity with the rest oft the world, on the one hand through Einstein's equation, on the other hand since it is Newton's gravitational constant which determines the intrinsic scale of the Planck medium. The Higgs contribution $T_{\mu \nu}^{\mathrm{vac}}$ is to be identified as a contribution to the classical ideal fluid energy-momentum tensor

$$
T_{\mu \nu}=(\rho+p) u_{\mu} u_{\nu}-p g_{\mu \nu}
$$

where $\rho$ is the density, $p$ the pressure, $u^{\mu} \equiv \mathrm{d} x^{\mu} / \mathrm{d} s$ is the contravariant four velocity of the fluid and $u_{\mu}=g_{\mu \nu} u^{\nu}$. The comparison shows that the Higgs implies a pressure

$$
p_{\mathrm{vac}}=p_{\phi}=-\rho_{\phi} .
$$

On the classical field level, assuming spatial isotropy (i.e. $\phi$ only depends on time), the Higgs contribution to energy density and pressure is given by

$$
\rho_{\phi}=\frac{1}{2} \dot{\phi}^{2}+V(\phi) ; \quad p_{\phi}=\frac{1}{2} \dot{\phi}^{2}-V(\phi) .
$$

The second Friedman equation has the form

$$
\ddot{a} / a=-\frac{\ell^{2}}{2}(\rho+3 p),
$$

where $\ell^{2}=8 \pi G / 3 . M_{\mathrm{Pl}}=(G)^{-1 / 2}$ is the Planck mass, $G$ is Newton's gravitational constant and for any quantity $X$ we denote time derivatives by $\dot{X}$. The condition for growth $\ddot{a}>0$, requires $p<-\rho / 3$ and hence $\frac{1}{2} \dot{\phi}^{2}<V(\phi)$. CMB observations strongly favor the slow-roll inflation $\frac{1}{2} \dot{\phi}^{2} \ll V(\phi)$ condition and hence the dark energy equation of state $w=p / \rho \simeq-1$. Indeed, the Planck mission measured $w=-1.13_{-0.10}^{+0.13}[60$. The first Friedman equation reads

$$
\dot{a}^{2} / a^{2}+k / a^{2}=\ell^{2} \rho,
$$

and may be written as $H^{2}=\ell^{2}\left[V(\phi)+\frac{1}{2} \dot{\phi}^{2}\right]=\ell^{2} \rho$. The kinetic term $\dot{\phi}^{2}$ is controlled by $\dot{H}=-\frac{3}{2} \ell^{2} \dot{\phi}^{2}$ related to the observationally controlled deceleration parameter $q(t)=-\ddot{a} a / \dot{a}^{2}$. In addition we have the field equation

$$
\ddot{\phi}+3 H \dot{\phi}=-V^{\prime}(\phi) \equiv-\mathrm{d} V(\phi) / \mathrm{d} \phi .
$$

It follows that the Higgs likely can be identified as the scalar field which drives inflation provided $\frac{1}{2} \dot{\phi}^{2} \ll V(\phi)$. It is precisely the quadratically enhanced mass term in the Higgs potential which makes the Higgs a good inflaton candidate. A dominant mass term also looks to imply the inflaton to represent essentially a free field. This seems to be supported by recent Planck mission constraints on non-Gaussianity [47. 
The amount of inflation is quantified by the inflation exponent $N_{e}$ given by

$$
\begin{aligned}
N_{e} & =\ln \frac{a\left(t_{\text {end }}\right)}{a\left(t_{\text {initial }}\right)}=\int_{t_{i}}^{t_{e}} H(t) \mathrm{d} t=\int_{\phi_{i}}^{\phi_{e}} \frac{H}{\dot{\phi}} \mathrm{d} \phi \\
& =-\frac{8 \pi}{M_{\mathrm{Pl}}^{2}} \int_{\phi_{i}}^{\phi_{e}} \frac{V}{V^{\prime}} \mathrm{d} \phi=H\left(t_{e}-t_{i}\right),
\end{aligned}
$$

where we have utilized the field equation $H \mathrm{~d} t=-H^{2} / V^{\prime} \mathrm{d} \phi$ and the first Friedman equation $H^{2}=\ell^{2} V$ in the slow-roll approximation. The times $t_{i}$ and $t_{e}$ denote beginning and end of inflation, where correspondingly the scalar field attains values $\phi_{i}$ and $\phi_{e}$, respectively. For $H=$ constant we would have $N_{e}=H\left(t_{e}-t_{i}\right)$, which is a good approximation to the extent that the total energy density $\rho_{\text {tot }} \simeq \rho_{\Lambda}$ is dominated by the cosmological constant (CC). In the symmetric phase $V / V^{\prime}>0$ and hence $\phi_{i}>\phi_{e}$. A rescaling of the potential does not affect inflation, but the relative weight of the terms is crucial. A precise analysis of the relative importance of the various possible components will be the main topic of the next Section. For the SM Higgs potential in the symmetric phase, denoting $z \equiv \frac{\lambda}{6 m^{2}}$, and a potential $V(\phi)=V(0)+\Delta V(\phi)$ we have a term $\frac{V(0)}{2 m^{2}} \frac{1}{\phi} \frac{1}{1+z \phi^{2}}$ plus $\frac{\Delta V}{V^{\prime}}=\frac{\phi}{4}\left(1+\frac{1}{1+z \phi^{2}}\right)$ and thus with

$\mathcal{I}=\int_{\phi_{e}}^{\phi_{i}} \frac{V}{V^{\prime}} \mathrm{d} \phi=\frac{V(0)}{2 m^{2}}\left[\ln \frac{\phi_{i}^{2}}{\phi_{e}^{2}}-\ln \frac{{\phi_{i}}^{2} z+1}{{\phi_{e}}^{2} z+1}\right]+\frac{1}{8}\left[\phi_{i}^{2}-\phi_{e}^{2}+\frac{1}{z} \ln \frac{{\phi_{i}}^{2} z+1}{\phi_{e}{ }^{2} z+1}\right]$

we obtain

$$
N_{e}=\frac{8 \pi}{M_{\mathrm{Pl}}^{2}} \mathcal{I}
$$

Below we will show that

$$
V(0)=\frac{m^{2}}{2}\left\langle 0\left|\phi^{2}\right| 0\right\rangle+\frac{\lambda}{24}\left\langle 0\left|\phi^{4}\right| 0\right\rangle
$$

like $m^{2}$ and $z=\frac{\lambda}{6 m^{2}}$ all are known SM quantities! $N_{e}$ large requires $\phi_{i} \gg \phi_{e}$. With $\phi_{i} \simeq 4.51 M_{\mathrm{Pl}}$, a value motivated by the amount of inflation wanted, and taking into account the running of parameters as given by the standard $\overline{\mathrm{MS}} \mathrm{RG}$, we find $\phi_{e} \simeq 2.01 \times 10^{-3} M_{\mathrm{Pl}}$ and $N_{e} \approx 64.68$ at the end of inflation at about $t \simeq 450 t_{\mathrm{Pl}}$, a value not far above the phenomenologically required minimum bound. $N_{e}$ may be increased by increasing $\phi_{i}$. Figure 2 gives an overview already of important features of the early inflation period.

As we will see, SM Higgs inflation is far from working obviously. The reason why SM inflation is quite tricky is the fact that the form of the potential is given and the parameters are known. What is at our disposal is essentially 

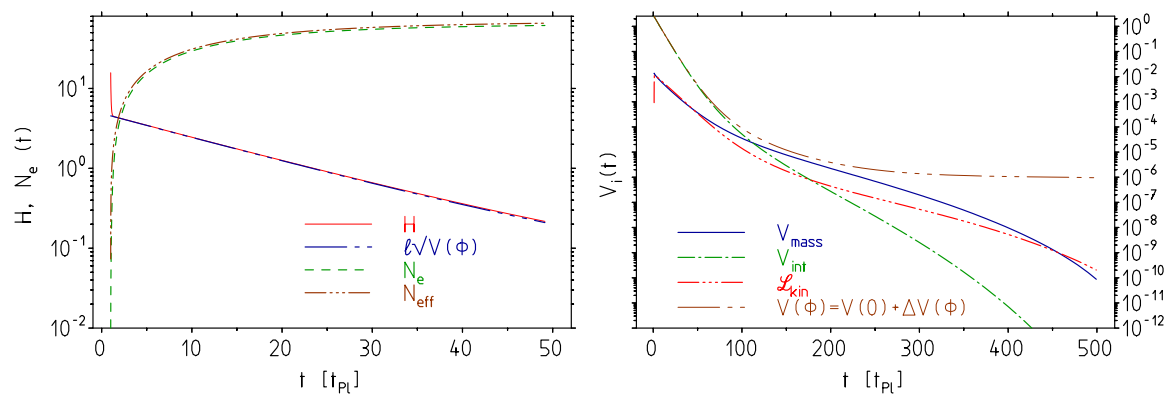

Figure 2: The inflation era. Left: $N_{e} \approx 66$ is reached at time $t \approx 50 t_{\mathrm{Pl}}$. The Hubble constant $H$ is satisfying $H \approx \ell \sqrt{V(\phi)}$ very well shortly after Planck time. The evaluation of $N_{e}$ via Eq. (21) agrees very well with the numerical $N_{\text {eff }}=\ln a(t) / a\left(t_{\mathrm{Pl}}\right)$ obtained by solving the coupled set of dynamical equations. Right: dark energy contributions during inflation. Shown are the separate terms of the Higgs potential together with the Higgs kinetic term. Slow-roll inflation stops at about $t \approx 450 t_{\mathrm{Pl}}$ when $\mathcal{L}_{\text {kin }} \sim V_{\text {mass }} \gg V_{\text {int }}$, after which the Higgs behaves as a damped quasi-free oscillating field.

only the value of the Higgs field at the Planck scale, since in the experimentally accessible low energy region the Higgs field is not an observable and we only know its vacuum expectation value.

In the following we are dealing with physics near the Planck scale, where the bare theory resides, and by $\phi, V(\phi), \lambda$ and $m$ we denote the bare quantities (fields and parameters), if not specified otherwise.

The papers is organized as follows: in Section 2 we study in some detail how Higgs inflation actually works. Section 3 is devoted to considerations of reheating and the possibility of SM baryogenesis. A solution of the cosmological constant problem of the SM is presented in Section 4. Conclusions are following in Section 5.

\section{The profile of Higgs inflation}

In Ref. [7] we have worked out the effective structure of the SM up to the Planck scale, by calculating the bare SM parameters in the $\overline{\mathrm{MS}}$ scheme as a function of the energy scale $\mu$. Above the Higgs transition scale $\mu_{0}$, in the early universe, the SM is in the unbroken phase, where four heavy Higgs fields exist besides the other highly relativistic degrees of freedom. The analysis is strongly supporting that the Higgs is actually responsible for the phenomenon of inflation. Here we present a more detailed investigation and perform consistency checks concerning the SM inflation scenario (see Ref. [48] and references therein). So far we have not said much about the size of the Higgs field and whether it is adequate to just check the parameters in the potential to decide about the relative importance of the different terms. Therefore some more details on the impact of the quadratic 
enhancement on the inflation profile are in order. We have shown that for a Higgs mass of about $126 \mathrm{GeV}$ there is a phase transition at a scale about $\mu_{0} \sim 1.4 \times 10^{16} \mathrm{GeV}$ and at temperatures above this scale the $\mathrm{SM}$ is in the symmetric phase in which the Higgs potential exhibits a huge bare mass term of size

$$
m^{2} \sim \delta m^{2} \simeq \frac{M_{\mathrm{Pl}}^{2}}{32 \pi^{2}} C\left(\mu=M_{\mathrm{Pl}}\right) \simeq\left(0.0295 M_{\mathrm{Pl}}\right)^{2},
$$

or $m^{2}\left(M_{\mathrm{Pl}}\right) / M_{\mathrm{Pl}}^{2} \approx 0.87 \times 10^{-3}$. For large slowly varying fields, the field equation of motion simplifies to the slow-roll equation $3 H \dot{\phi} \approx-V^{\prime}$ with $H \approx \ell \sqrt{V}$, which describes a decay of the field.

In the symmetric phase the key object of interest is the SM Higgs potential $V(\Phi)=m^{2} \Phi^{+} \Phi+\frac{\lambda}{3 !}\left(\Phi^{+} \Phi\right)^{2}$ eventually dominated by the mass term $m^{2} \Phi^{+} \Phi$. Here $\Phi$ is the complex SM $S U(2)$ Higgs doublet field, which in the symmetric phase includes four heavy physical scalars:

$$
\Phi=\left(\begin{array}{c}
\phi^{+} \\
\phi_{0}
\end{array}\right) ; \phi^{+}=\mathrm{i} \frac{\phi_{1}-\mathrm{i} \phi_{2}}{\sqrt{2}}, \phi_{0}=\frac{H-\mathrm{i} \phi}{\sqrt{2}}, \phi=\phi_{3}
$$

in terms of the real fields $H, \phi_{i},(i=1,2,3)$. In the broken phase $\langle 0|H| 0\rangle=v$, the $\phi_{i}$ 's transmute to gauge degrees of freedom and we get the Higgs potential $V(H)=\frac{m^{2}}{2} H^{2}+\frac{\lambda}{24} H^{4}$, considered so far. We adopt the "would be" charge assignments, as they manifest themselves in the broken phase. In the symmetric phase $U(1)_{\mathrm{em}}$ is not yet singled out and there are no photons and in place of charge and flavor there are the gauge symmetry assignments only, the singlets, doublets and triplets. Still hyper-charge $U(1)_{Y}$ is conserved. We will nevertheless use field assignments as if we would be in the broken phase.

There are two quantities, which we cannot get by just extrapolating the SM beyond the Higgs transition point. One is the renormalized mass $m$ in the symmetric phase, the other is the magnitude of the Higgs field. We assume here that the renormalized $m^{2}$ is small relative to $\delta m^{2}$, which we can calculate. The Higgs field in the LEESM only depends logarithmically on the cutoff, such that we naturally expect the field the by small in the sense $m^{2} \gg\left\langle 0\left|\Phi^{+} \Phi\right| 0\right\rangle$. In fact the Higgs fields must be very heavy as well in order that the Higgs can be the inflaton. Here, the field equation and the Friedman equations actually help to estimate the proper initial value of the field. At least in the slow-roll inflation scenario we know that the Higgs field has to decay fast. Obviously, large Higgs fields work against Gaussianity, which requires the dominance of the Higgs mass term, and hence

$$
\phi^{2}<\frac{12 m^{2}}{\lambda}=\frac{3 C}{8 \pi^{2} \lambda} M_{\mathrm{Pl}}^{2}=\frac{3\left(2 \lambda+\frac{3}{2} g^{\prime 2}+\frac{9}{2} g^{2}-12 y_{t}^{2}\right)}{8 \pi^{2} \lambda} M_{\mathrm{Pl}}^{2}
$$

during inflation. With our input parameters, mass term dominance holds when

$$
|\phi| \ll \sqrt{12 / \lambda} m\left(M_{\mathrm{Pl}}\right) \approx 0.2726 M_{\mathrm{Pl}} .
$$

Since $\phi$ is decreasing rapidly during inflation, the condition of Gaussianity gets dynamically established at some point, at which however the dark energy density 
$V(\phi)$ has to be large enough to keep inflation going. We also note that RG evolution may yield a substantially smaller value for $\lambda\left(M_{\mathrm{Pl}}\right)$ in case $y_{t}\left(M_{t}\right)$ would be slightly larger than our estimate (see Table 1). If $\lambda\left(M_{\mathrm{Pl}}\right)=0$, the minimum value allowed for our scenario to work, we have $C(\mu) \leq 0$ for $y_{t}\left(M_{\mathrm{Pl}}\right)>0.353$, and there is no Higgs transition below $M_{\mathrm{Pl}}$. Thus, for our scenario to work we need $y_{t}\left(M_{\mathrm{Pl}}\right)<0.353$, given the gauge couplings at $M_{\mathrm{Pl}}$.

In any case, it is very interesting that the whole scenario based on the existence of the Higgs phase transition sufficiently below the Planck scale, requires a window in parameter space which is very close to whatever SM parameters estimates yield. Can this be an accident? As our SM inflation scenario is supported by CMB data, we expect that at the end something close to our scenario should turn out to describe reality.

In the very early universe radiation is dominating the scene, this is not changed by a large cosmological constant term, even the curvature term may win over the cosmological constant close enough to the Planck time. The Hubble constant in our scenario, in the symmetric phase, during the radiation dominated era is given by $H=\ell \sqrt{\rho} \simeq 1.66\left(k_{B} T\right)^{2} \sqrt{102.75} M_{\mathrm{Pl}}^{-1}$ such that at Planck time $H_{i} \simeq 16.83 M_{\mathrm{Pl}}$. One expects that $V(\phi)$ does not exceed too much a possible vacuum energy of size $M_{\mathrm{Pl}}^{4}$. The condition $V\left(\phi_{0}\right)=M_{\mathrm{Pl}}^{4}$ yields an initial value $\phi_{0}$ as follows: with $a=6 m^{2} / \lambda, b=24 M_{\mathrm{Pl}}^{4} / \lambda$ and $r=\sqrt{b+a^{2}}$ we find

$$
\phi_{0}=\sqrt{r-a} \simeq 4.40 \times 10^{19} \mathrm{GeV}=3.61 M_{\mathrm{Pl}} .
$$

It is well known that the CMB horizon problem requires an inflation index $N_{e}>$ 60. This index may be considered as a direct measure of the unknown initial value $\phi_{0}$, and hence observational inflation data actually provides a lower bound on this input. With the plausible estimate (27) we actually obtain $N_{e} \sim 57$ but we easily can reach an index above $N_{e} \sim 60$ by slightly increasing (27). We will adopt an initial field enhanced by $25 \%$ i.e. as our standard input we choose

$$
\phi_{0}=4.51 M_{\mathrm{Pl}} \text {. }
$$

Notice that the dominance of the mass term at $M_{\mathrm{Pl}}$ would require the condition (26). The assumption here is that the Higgs field, having dimension one, in the cutoff system with intrinsic scale $M_{\mathrm{Pl}}$ should naturally be $O\left(M_{\mathrm{Pl}}\right)$. In fact we assume that the Friedman equations as well as the Higgs field equations start to be valid after $t_{\mathrm{Pl}}$ only, which does not mean that at earlier times temperatures $T>T_{\mathrm{Pl}}$ and corresponding excitations of the system are not expected to exist. They exist in any case. As the field decays exponentially at first, when $V_{\text {int }} \gg V_{\text {mass }}$, during early inflation (see Fig. 2 and Fig. 6 below) the mass term dominance (free massive field as an inflaton) is reached in any case during inflation at times $t \gtrsim 150 t_{\mathrm{Pl}}$. Once $V_{\text {mass }} \gg V_{\text {int }}$, the field continues to decay exponentially, because of the vacuum term $V(0)$, which largely determines the Hubble rate $H$.

In order to get an idea on how the different density components affect the evolution at very early times we follow Refs. 49,50 . Let us rewrite the $1^{\text {st }}$ and 
$2^{\text {nd }}$ Friedman equations in the form

$$
\left(\frac{\dot{a}}{a}\right)^{2}=\ell^{2}\left(\rho_{\gamma}+\rho_{\Lambda}-\rho_{k}\right) \quad \text { and } \quad \frac{\ddot{a}}{a}=\ell^{2}\left(-\rho_{\gamma}+\rho_{\Lambda}\right),
$$

where

$$
\rho_{\gamma}=\rho_{\gamma i}\left(\frac{a_{i}}{a}\right)^{4} ; \rho_{\Lambda}=\frac{\Lambda}{3 \ell^{2}} ; \rho_{k}=\frac{k c^{2}}{\ell^{2} a^{2}},
$$

represent the radiation, cosmological constant $\Lambda$ and curvature contribution to the energy densities, respectively. Indexed by $i$ are the corresponding initial quantities at the Planck scale. Matter will be produced by reheating and at the EW phase transition at a later stage. By adding the two Friedman equations one obtains

$$
\frac{1}{2} \frac{\mathrm{d}^{2}}{\mathrm{~d} t^{2}} a^{2}(t)=2 \ell^{2} \rho_{\Lambda} a^{2}-k c^{2},
$$

which may be written as

$$
\ddot{X}=E_{\Lambda}^{2} X ; \quad X \equiv a^{2}-2 k c^{2} t_{\Lambda}^{2} ; \quad E_{\Lambda}=2 \ell \sqrt{\rho_{\Lambda}}=1 / t_{\Lambda},
$$

with solution

$$
a(t)=\left(c_{1} \mathrm{e}^{-t E_{\Lambda}}+\frac{1}{2} c_{2} \mathrm{e}^{t E_{\Lambda}}+2 k c^{2} t_{\Lambda}^{2}\right)^{1 / 2} .
$$

The integration constants $c_{1}$ and $c_{2}$ may be fixed by assuming special initial values for $a_{i}=a\left(t_{i}\right)$ and $H_{i}=\dot{a}_{i} / a_{i}$ at the initial time $t_{i}$, which we choose to be the Planck time. One obtains

$c_{1}=\frac{1}{2}\left(a_{i}^{2}-2 \dot{a}_{i} a_{i} t_{\Lambda}-2 k c^{2} t_{\Lambda}^{2}\right) \mathrm{e}^{t_{i} E_{\Lambda}} ; \quad c_{2}=\left(a_{i}^{2}+2 \dot{a}_{i} a_{i} t_{\Lambda}-2 k c^{2} t_{\Lambda}^{2}\right) \mathrm{e}^{-t_{i} E_{\Lambda}}$,

where $\dot{a}_{i}$ can be calculated in terms of the initial densities $\rho_{\gamma i}, \rho_{k i}$ and $\rho_{\Lambda}$, which yields

$$
\left(2 t_{\Lambda} \dot{a} a\right)_{i}=a_{i}^{2}\left(\frac{\rho_{\gamma i}}{\rho_{\Lambda}}-\frac{\rho_{k i}}{\rho_{\Lambda}}+1\right)^{1 / 2} ; 2 k c^{2} t_{\Lambda}^{2}=\frac{1}{2} a_{i}^{2} \frac{\rho_{k i}}{\rho_{\Lambda}} .
$$

The solution then reads

$$
a(t)=a_{i}\left(\cosh \tau+\frac{1}{2} \frac{\rho_{k i}}{\rho_{\Lambda}}(1-\cosh \tau)+\left(\frac{\rho_{\gamma i}}{\rho_{\Lambda}}-\frac{\rho_{k i}}{\rho_{\Lambda}}+1\right)^{1 / 2} \sinh \tau\right)^{1 / 2}
$$

where $\tau=\left(t-t_{i}\right) E_{\Lambda}$ is the reduced time. The Hubble function is then

$$
H(t)=\frac{E_{\Lambda}}{2}\left(\frac{a_{i}}{a}\right)^{2}\left(\left(1-\frac{1}{2} \frac{\rho_{k i}}{\rho_{\Lambda}}\right) \sinh \tau+\left(\frac{\rho_{\gamma i}}{\rho_{\Lambda}}-\frac{\rho_{k i}}{\rho_{\Lambda}}+1\right)^{1 / 2} \cosh \tau\right),
$$



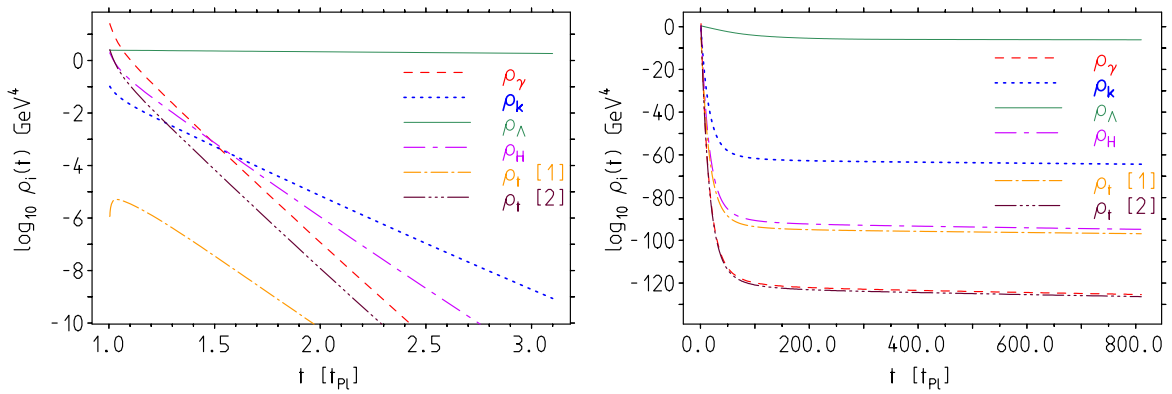

Figure 3: Right: this graph shows that the dark energy term as given by (1) is dominating the density quite soon after the big bang, and thus indeed is driving inflation. Left: analogous plot taking into account the Higgs decay into $t \bar{t}[1]$ up to before the Higgs transition $\left(\phi_{0} \simeq 4.5 M_{\mathrm{Pl}}\right) . \rho_{t}[2]$ (in the plot little below $\rho_{\gamma}$ ) is the top component in $\rho_{\gamma}$, which denote the total radiation density without the reheating part. For later reference we also show the top quark density $\rho_{t}$ obtained from reheating [1] in comparison with the fraction of top quark radiation which is part of $\rho_{\gamma}$. Included is also the non-relativistic heavy Higgs component $\rho_{H}$ given by Eq. (51).

while the acceleration reads

$$
\frac{\ddot{a}}{a}=\frac{E_{\Lambda}^{2}}{2}\left(1-\frac{1}{2} \frac{\rho_{k i}}{\rho_{\Lambda}}\left(\frac{a_{i}}{a}\right)^{2}\right)-H^{2} .
$$

We can then calculate the evolution of the various components which we show in Fig. 3 together with the top quark density and the heavy Higgs density to be introduced later.

As an initial value for the Higgs field at $M_{\mathrm{Pl}}$ we adopt (28). In general, if the initial value of $\phi$ is exceeding about $\frac{1}{5} M_{\mathrm{Pl}}$ one can neglect $\ddot{\phi}$ in the field equation as well as the kinetic term $\frac{1}{2} \dot{\phi}^{2}$ in the Friedman equation. We expect inflation to start at Planck time $t_{i} \equiv t_{\text {initial }}=t_{\mathrm{Pl}} \simeq 5.4 \times 10^{-44} \mathrm{sec}$ and to stop definitely at $t_{\mathrm{CC}} \simeq 2.1 \times 10^{-40} \mathrm{sec}$ the at drop of the CC to be discussed in Sec. 4. As mentioned earlier the efficient era of slow-roll inflation ends at about $t \simeq 450 t_{\mathrm{Pl}}$. We adopt, somewhat arbitrary, a period including the bare Higgs transition point at $t_{e} \equiv t_{\mathrm{end}}=t_{\mathrm{Higgs}} \approx 4.7 \times 10^{-41} \mathrm{sec}$. As $m$ is substantially lower than $M_{\mathrm{Pl}}$ actually for strong fields the interaction term is dominating. Then $\phi$ decays exponentially like

$$
\phi(t)=\phi_{0} \mathrm{e}^{-E_{0}\left(t-t_{0}\right)} ; E_{0}=\frac{\sqrt{2 \lambda}}{3 \sqrt{3} \ell} \approx 4.3 \times 10^{17} \mathrm{GeV} ; V_{\text {int }} \gg V_{\text {mass }},
$$

while a dominant mass term leads to a decay linear in time

$$
\phi(t)=\phi_{0}-X_{0}\left(t-t_{0}\right) ; X_{0}=\frac{\sqrt{2} m}{3 \ell} \approx 7.2 \times 10^{35} \mathrm{GeV}^{2} ; V_{\text {mass }} \gg V_{\text {int }} .
$$


However, this is not quite what corresponds to the true SM prediction. As we will argue below we have to account for a cosmological constant term $V(0) \equiv$ $\langle V(\phi)\rangle$, which in the field equation contributes the Hubble constant as $H \simeq$ $\ell \sqrt{V(0)+\Delta V(\phi)}$. At the begin of inflation $V(0)$ is of size comparable to $V_{\text {int }}$ while later on the mass term starts to dominate over the interaction term, but still $V(0) \gg V_{\text {mass }}$, such that actually also during this era we have an exponential decay

$$
\phi(t) \approx \phi_{0} \mathrm{e}^{-E_{0}\left(t-t_{0}\right)} ; E_{0} \approx \frac{m^{2}}{3 \ell \sqrt{V(0)}} \approx 6.6 \times 10^{17} \mathrm{GeV} ; V_{\text {mass }} \gg V_{\text {int }}
$$

of the field. Thus in any case, during slow-roll inflation, the decay of the dynamical part of the Higgs field is exponential at dramatic rate. Actually, as we will see, the cosmological constant proportional to $\rho_{\Lambda}=V(\phi) \approx V(0)$ and a corresponding Hubble constant $H \approx \ell \sqrt{V(0)}$ long after slow-roll inflation has ended, will decrease dramatically when $V(0)$ drops essentially to zero at a scale $\mu_{\mathrm{CC}} \simeq 5.0 \times 10^{15} \mathrm{GeV}$. Without the contribution $V(0)$, the fast decay of the Higgs field could be in contradiction with the observationally favored slow-roll scenario. An important point here is that the Higgs potential has a calculable non-vanishing vacuum expectation value in the bare system. Vacuum contractions are ruled by Wick ordering?: $\phi^{2}=\left\langle\phi^{2}\right\rangle+{\phi^{\prime}}^{2}$ and $\phi^{4}=\left\langle\phi^{4}\right\rangle+3 !\left\langle\phi^{2}\right\rangle{\phi^{\prime}}^{2}+{\phi^{\prime}}^{4}$ which yields a constant $V(0)=\langle V(\phi)\rangle$ and a mass shift $m^{\prime 2}=m^{2}+\frac{\lambda}{2}\left\langle\phi^{2}\right\rangle$ plus the potential in terms of the fluctuation field $\Delta V(\phi)$. Note that in SM notation $\left\langle 0\left|\Phi^{+} \Phi\right| 0\right\rangle=\frac{1}{2}\left\langle 0\left|H^{2}\right| 0\right\rangle \equiv \frac{1}{2} \Xi$ is a singlet contribution. We thus obtain a quasi-constant vacuum density

$$
V(0)=\frac{m^{2}}{2} \Xi+\frac{\lambda}{8} \Xi^{2} ; \Xi=\frac{M_{\mathrm{Pl}}^{2}}{16 \pi^{2}},
$$

the VEV of the potential, which does contribute to the cosmological constant. The field equation, which only involves the time dependent part, is affected via a modyfied Hubble constant and the shifted effective mass (see below). The $Z_{2}$ symmetry $\Phi \rightarrow-\Phi$ and the SM gauge symmetry remain untouched. The $\Phi^{+} \Phi$ VEV $\frac{1}{2} \Xi$ in principle should be calculable in a lattice SM. One has to be aware of course that we do not know the true underling Planck ether system. Such estimates in any case would be instructive in understanding underlying mechanisms. Since, as a result of the SM RG evolution, effective SM parameters are well within the perturbative regime one can actually calculate $\Xi$. In leading order we just have Higgs self-loops

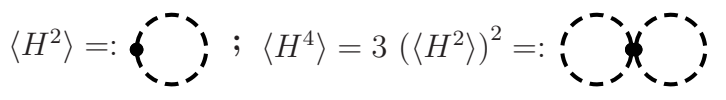

\footnotetext{
${ }^{2}$ Wick ordering amounts to a redefinition of the operator basis by subtracting c-number self contractions of the fields. In gauge theories one is advised not to express the Lagrangian in terms of Wick ordered fields because the originally manifest symmetry would be mixed up and become intransparent. Wick ordering in general is not just equivalent to subtracting the VEV of the Lagrangian.
} 

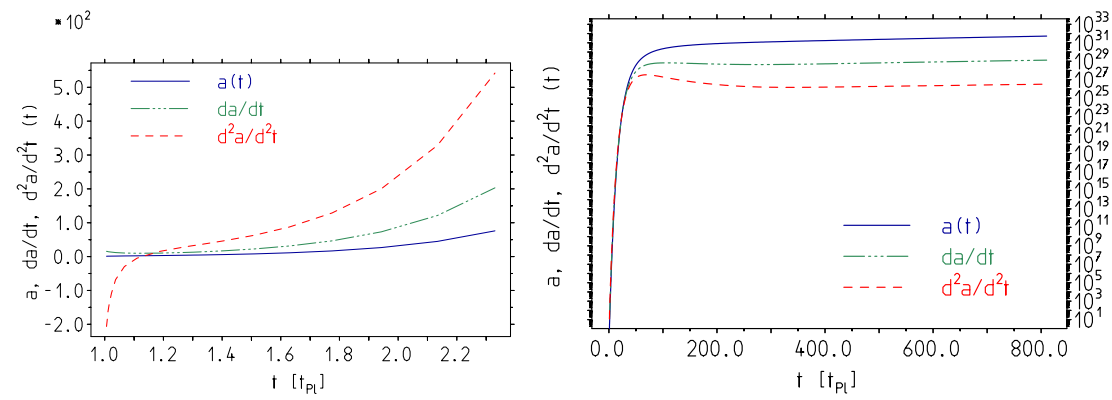

Figure 4: The FRW radius and its derivatives for $k=1$ as a function of time all in units of the Planck mass, i.e. for $M_{\mathrm{Pl}}=1$. Left: the start. Right: the expansion before the Higgs transition.

given by $\Xi=\left\langle H^{2}\right\rangle=\frac{\Lambda^{2}}{16 \pi^{2}} 3$. Again, for the fluctuation field, which decays exponentially, in the early phase of inflation we adopt the initial value $\phi_{0} \approx$ $4.51 M_{\mathrm{Pl}}$ estimated above in Eq. (28). In the potential of the fluctuation field and the corresponding field equation the mass square now is given by

$$
m^{\prime 2}=m^{2}+\frac{\lambda}{2} \Xi
$$

This actually is a very interesting shift as it modifies the Higgs transition point to lower values with new effective coefficient

$$
C_{1}^{\prime}=C_{1}+\lambda=3 \lambda+\frac{3}{2} g^{\prime 2}+\frac{9}{2} g^{2}-12 y_{t}^{2}
$$

For our values of the $\overline{\mathrm{MS}}$ input parameters, we obtains (see Fig. 13 below)

$$
\mu_{0} \approx 1.4 \times 10^{16} \mathrm{GeV} \rightarrow \mu_{0}^{\prime} \approx 7.7 \times 10^{14} \mathrm{GeV},
$$

as a relocation of the Higgs transition point.

We can now solve the coupled system of equations (29)30 and (19) numerically e.g. by the Runge-Kutta method. It adds to the above analytic solution for a constant "cosmological constant" the Higgs field dynamics. Results for the FRW radius $a(t)$ and the field $\phi(t)$ together with the derivatives are displayed in Figs. 45] Figure 6] shows how the different terms of the bare Lagrangian evolve. At later inflation times the mass term is dominating as originally expected, but the dominance is not very pronounced. The temporary mass term dominance is important for the observed Gaussianity by the Planck mission [9]. At about $t \simeq 450 t_{\mathrm{Pl}}$ slow-roll inflation ends and free field oscillations begin. The two panels illustrate the difference obtained between working with running couplings vs. keeping couplings fixed as given at the Planck scale. It turns out to

\footnotetext{
${ }^{3}$ Including the heavy Higgs mass effect we have $\Xi=\frac{M_{\mathrm{Pl}}^{2}}{16 \pi^{2}}\left(1-\frac{m^{2}}{M_{\mathrm{Pl}}^{2}} \ln \left(\frac{M_{\mathrm{P} 1}^{2}}{m^{2}}+1\right)\right)$. This mass correction is negligible here.
} 

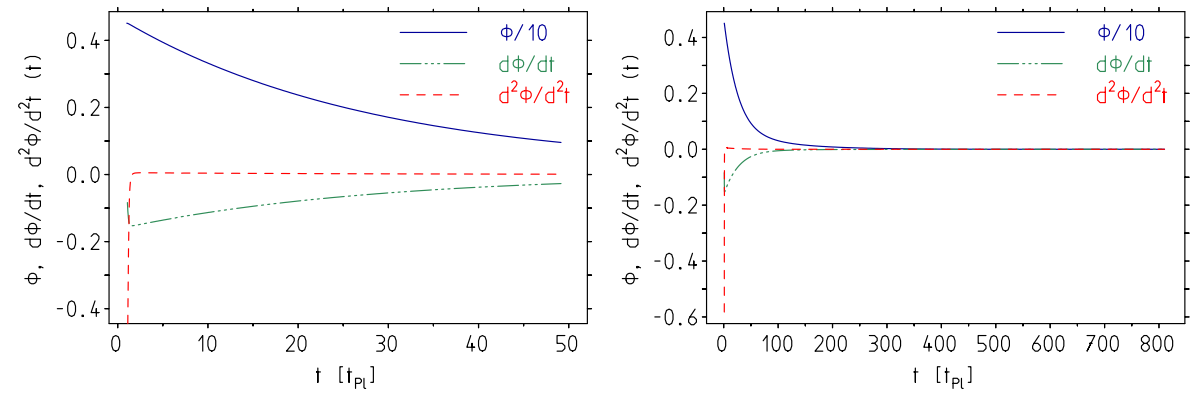

Figure 5: The Higgs field and its derivatives for $k=1$ as a function of time all in units of the Planck mass. Left: the Higgs field at start. Right: the Higgs field decay before the Higgs transition. The field starts oscillating strongly like a free field once $\mathcal{L}_{\text {kin }} \sim V_{\text {mass }}$ while $V_{\text {int }} \ll V_{\text {mass }}$ (see Fig. 6).
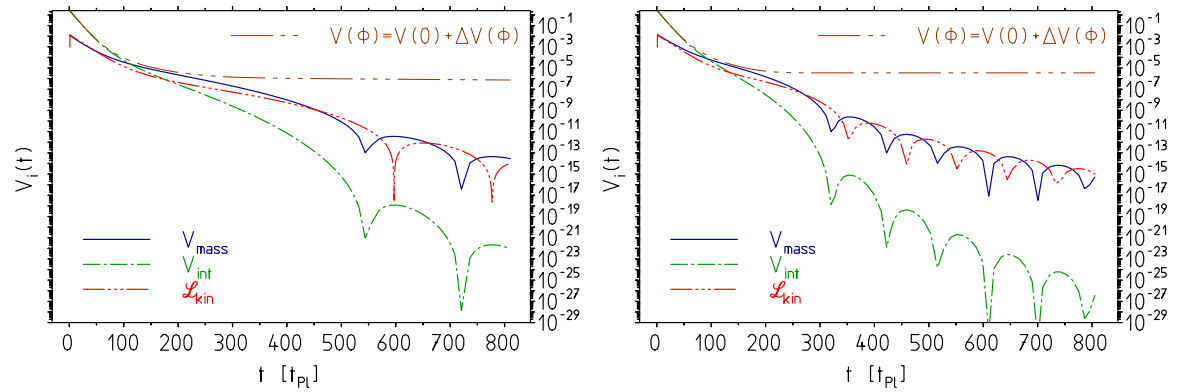

Figure 6: The mass-, interaction- and kinetic-term of the bare Lagrangian in units of $M_{\mathrm{Pl}}^{4}$ as a function of time. Left: the relative contributions when proper running of SM couplings are taken into account. The mass term is dominating in the range $t \simeq 100$ to $450 t_{\mathrm{Pl}}$, where the slow-roll era ends and damped quasi-free field oscillations start. Right: fake solution based on constant couplings. 
be crucial to take into account the scale dependence of the coupling, throughout the calculation. How can it be that the minor changes in SM couplings between $M_{\mathrm{Pl}}$ and $\mu_{0}$ make up such dramatic difference? The reason is quite simple, the effects are enhanced by the quadratic "divergence" enhancement factor $\frac{M_{\mathrm{Pl}}^{2}}{32 \pi^{2}}$, which actually makes the whole thing work. One of the key criteria during the inflation era is the validity of the dark energy equation of state $w=p / \rho=-1$, which we display in Fig. 7 as a function of time before the bare Higgs transition point $\mu_{0}$. In fact $w=-1$ is perfectly satisfied quite early after Planck time. This shows how dark energy is supplied by the Higgs system.

The slow-roll criteria are usually tested by the coefficients

$$
\varepsilon \equiv \frac{M_{\mathrm{Pl}}^{2}}{8 \pi} \frac{1}{2}\left(\frac{V^{\prime}}{V}\right)^{2} ; \eta \equiv \frac{M_{\mathrm{Pl}}^{2}}{8 \pi} \frac{V^{\prime \prime}}{V}
$$

where $\varepsilon \ll 1$ ensures $p \simeq-\rho$, while $\varepsilon, \eta \ll 1$ ensure slow-roll for a long enough time, maintaining $\ddot{\phi} \ll 3 H \dot{\phi}$. When slow-roll ends, $\phi$ oscillates rapidly about $\phi=0$ and the oscillations lead to abundant particle production which is reheating the universe. More on this below.

Actually, as we will see, the condition $\eta \ll 1$ is a sufficient condition only and not a necessary one. For a SM Higgs type potential especially $\eta \ll 1$ is hard to satisfy as we will explain below. In the LEESM scenario $V(0)$ plays a crucial role and actually keeps inflation going on in spite of the fact that the fluctuation field $\phi(t)$ is exponentially decaying. What stops the period of efficient slow-roll inflation is the decay of the field in the presence of a dominant quasi-constant $V(0)$.

For our set of parameters we indeed find inflation to work, and we obtain $N_{e} \sim 65$, essentially the required $N_{e}>60$. At this stage of our investigation we consider our results very promising as we have not yet exploited the substantial uncertainties in our key parameters $C_{1}\left(M_{\mathrm{Pl}}\right)$ and $\lambda\left(M_{\mathrm{Pl}}\right)$, which are to a large extent determined by the $\overline{\mathrm{MS}}$ input parameters $\lambda\left(M_{H}\right)$ and $y_{t}\left(M_{t}\right)$. The precise value of the latter, as we know, is somewhat controversial. In any case it is remarkable that such a scenario at worst is very close to what SM input parameters tell us. The estimates of the uncertainties and the evaluation of the spot in parameter space which supports our Higgs inflation scenario will be investigated in a forthcoming analysis. Figure 8 shows how well slow-roll inflation criteria are satisfied. For our numerical solution we obtain $\varepsilon \approx 2.3 \times 10^{-2}$ and $\eta \approx 21.3$, when slow-roll inflation ends at about $t \approx 450 t_{\mathrm{Pl}}$. As we learn from Fig. 8 as well as by inspection of the formal SM expressions, the indices $\varepsilon, \eta$ and the resulting spectral index $n_{S}=1-6 \varepsilon+2 \eta$ are extremely sensitive to the effective SM parameters, and in fact $\eta$, and consequently $n_{S}$, acquires values completely out of what formal inflation requirements suggest. In fact these results depend sensitively on the value of the Higgs field at the end of the inflation era. How do these indices depend on the SM parameters?

By $X$ we denote the rescaled $\phi^{2}$ field, $X=\frac{\phi^{2}}{\Xi}=16 \pi^{2} \frac{\phi^{2}}{M_{\mathrm{Pl}}^{2}}$, and by $\mu_{m}$ the unknown renormalized mass square, $\mu_{m}=\frac{2 m^{2}}{\Xi}$, in the following. For definiteness we take $m^{2}=m_{\phi}^{2}$ from Eq.(91). This choice barely affects the results as 


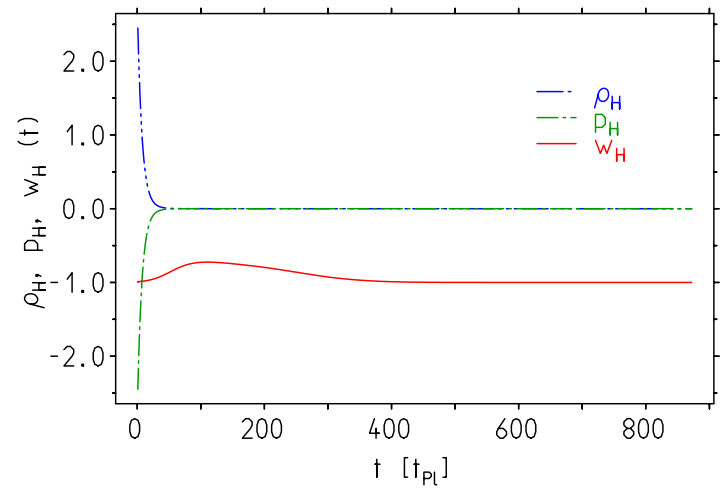

Figure 7: Higgs field density, pressure and equation of state. The Higgs provides dark energy beyond the inflation period which ends at about $t \simeq 450 t_{\mathrm{Pl}}$.
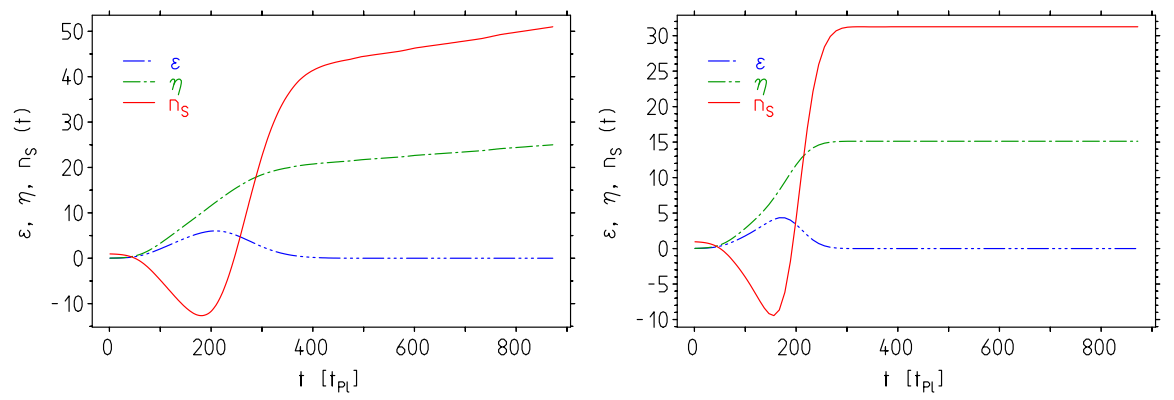

Figure 8: The slow-roll coefficients $\varepsilon, \eta$ and the spectral index $n_{S}=1-6 \varepsilon+2 \eta$ for the scalar fluctuations, as a function of time before the bare Higgs transition point. Left: correct result taking into account the running couplings. Right: fake results assuming fixed couplings as given at the Plank scale. 
long as we satisfy $m^{2} \ll \delta m^{2}$. For the Higgs potential we obtain

$$
\varepsilon=16 \pi X\left[\frac{3\left(\mu_{m}+C+\lambda\right)+\lambda X}{3\left(2\left(\mu_{m}+C\right)+\lambda\right)+6\left(\mu_{m}+C+\lambda\right) X+\lambda X^{2}}\right]^{2}
$$

such that for $\phi^{2} \rightarrow 0$ (late inflation)

$$
\varepsilon=16 \pi X\left[\frac{\mu_{m}+C+\lambda}{\left(2\left(\mu_{m}+C\right)+\lambda\right)}\right]^{2} \approx\left\{\begin{array}{ccc}
64 \pi^{3} \frac{\phi^{2}}{M_{\mathrm{P} 1}^{2}} & \text { for } \quad \mu_{m}+C \gg \lambda \\
256 \pi^{3} \frac{\phi^{2}}{M_{\mathrm{Pl}}^{2}} & \text { for } \quad \mu_{m}+C \ll \lambda
\end{array} .\right.
$$

At inflation begin $X \gg \mu_{m}+C, \lambda\left(\phi^{2}\right.$ large $)$ we have

$$
\varepsilon \sim \frac{1}{\pi} \frac{M_{\mathrm{Pl}}^{2}}{\phi^{2}}
$$

It is obvious that during inflation $\varepsilon$ is naturally expected to be small and in the limiting cases is independent of any SM parameters.

The second index

$$
\eta=24 \pi \frac{\mu_{m}+C+\lambda+\lambda X}{3\left(2\left(\mu_{m}+C\right)+\lambda\right)+6\left(\mu_{m}+C+\lambda\right) X+\lambda X^{2}}
$$

behaves differently. For $\phi^{2} \rightarrow 0$ (late inflation)

$$
\eta=8 \pi \frac{\mu_{m}+C+\lambda}{2\left(\mu_{m}+C\right)+\lambda} \approx\left\{\begin{array}{lll}
4 \pi & \text { for } & \mu_{m}+C \gg \lambda \\
8 \pi & \text { for } & \mu_{m}+C \ll \lambda
\end{array} .\right.
$$

So the SM predicts very large values when the field gets small towards the end of inflation. If an inflation criterion $\eta \ll 1$, or even $\eta<3 \varepsilon$ in case we require $n_{S}=1-6 \varepsilon+2 \eta<1$, would be a true necessary condition this would rule out the SM Higgs as a inflaton. At the beginning of inflation $X \gg C, \lambda\left(\phi^{2}\right.$ large $)$ we have

$$
\eta \sim \frac{3}{2 \pi} \frac{M_{\mathrm{Pl}}^{2}}{\phi^{2}} .
$$

A small $\phi^{2}$ makes $\varepsilon \sim \phi^{2} / M_{\mathrm{Pl}}^{2}$ in any case small, while a small $\eta$ requires $\phi$ not too small, as for $\phi \rightarrow 0 \eta$ takes large values depending on whether the mass or the interaction term of the potential dominates. As soon as one of the terms in the Lagrangian dominates, inflation is insensitive to $m^{2}$ or $\lambda$, respectively, as a rescaling of the potential is not affecting inflation. When $\lambda \rightarrow 0: \eta \rightarrow 4 \pi$; when $m^{2} \rightarrow 0: \eta \rightarrow 8 \pi$. This is what we observe in Fig. 8. Note that these results seem to be universal for a $\phi^{4}$ scalar potential and insofar are not specific for the Higgs sector with its particular parameters. However, at look at Eqs. (34) and (36) tells us that SM parameters have a strong impact on the decay rate of the scalar fluctuation field. The fact that with our educated guess for the initial value $\phi_{0}$ at $M_{\mathrm{Pl}}$ we obtain $\varepsilon$ very small while $\eta$ is obtained too large is a direct consequence of the fact that the field $\phi_{e}$ at late inflation times is 
obtained to be so small, which, however, strongly depends on the values of $m$ and/or $\lambda$ and hence on specific inputs $\lambda\left(M_{H}\right)$ and $y_{t}\left(M_{t}\right)$. Because of the strong decay of the field an increase of $\phi_{0}$ essentially does not affect $\eta$ at late inflaton times. This does not imply that we cannot get a sufficiently large inflation factor $N_{e}$, fortunately. The problem appears to be the spectral index for the scalar perturbations $n_{S}=1-6 \varepsilon+2 \eta$, to be considered next, which is constrained by observation to be $n_{S}<1$ requiring $\eta \leq 3 \varepsilon$, which seems to be very hard to satisfy for any symmetric Higgs type potential.

The indices just considered play a role in estimates of the scalar density fluctuations $\delta \rho=\frac{\mathrm{d} V}{\mathrm{~d} \phi} \delta \phi$, which are tailored by inflation [42,44 and exhibit a spectrum

$$
\begin{aligned}
A_{S}(k) & =\left.\frac{128 \pi}{3} \frac{V^{3}}{M_{\mathrm{Pl}}^{6}\left(V^{\prime}\right)^{2}}\right|_{k=a H} \\
& =\frac{\pi}{9\left(16 \pi^{2}\right)^{3}} \frac{\left(3\left(2\left(\mu_{m}+C\right)+\lambda\right)+6\left(\mu_{m}+C+\lambda\right) X+\lambda X^{2}\right)^{3}}{X\left(3\left(\mu_{m}+C+\lambda\right)+\lambda X\right)^{2}}
\end{aligned}
$$

to be evaluated at the moment when the physical scale of the perturbation $\lambda=a / k$ is equal to the Hubble radius $H^{-1}$ and thus at the event horizon. For small fields $X \rightarrow 0$ we have

$$
\begin{aligned}
A_{S}(k) & \sim \frac{\pi}{3\left(16 \pi^{2}\right)^{3}} \frac{1}{X} \frac{\left(2\left(\mu_{m}+C\right)+\lambda\right)^{3}}{\left(\mu_{m}+C+\lambda\right)^{2}} \\
& \sim \begin{cases}\frac{8 \pi}{3\left(16 \pi^{2}\right)^{4}}\left(\mu_{m}+C\right) \frac{M_{\mathrm{P} 1}^{2}}{\phi^{2}} ; & \mu_{m}+C \gg \lambda \\
\frac{\pi}{3\left(16 \pi^{2}\right)^{4}} \lambda \frac{M_{\mathrm{P} 1}^{2}}{\phi^{2}} & \mu_{m}+C \ll \lambda\end{cases}
\end{aligned}
$$

while for large fields $X \gg C, \lambda$ we find the behavior

$$
A_{S}(k) \sim \frac{\pi}{9} \lambda \frac{\phi^{6}}{M_{\mathrm{Pl}}^{6}} .
$$

Observations are parametrized by a power spectrum $A_{S}(k) \propto k^{n_{S}-1}$ where $n_{S}=1-6 \varepsilon+2 \eta$. The last relation is obtained by assuming that the Higgs potential dominates the scene and that a change of $k$ is given solely by a change of $\phi$ :

$$
\frac{\mathrm{d}}{\mathrm{d} \ln k}=-\frac{V^{\prime}}{2 H^{2}} \frac{\mathrm{d}}{\mathrm{d} \phi}
$$

in the slow-roll limit. The latter relation explains how $V^{\prime \prime}$ and herewith $\eta$ come into play. However, for the SM, given the fairly large quasi cosmological constant $V(0)$, which is largely determining the Hubble constant $H$ during inflation, the above relation and therefore the relation $n_{S}=1-6 \varepsilon+2 \eta$ does not apply. We therefore prefer to extract $n_{S}$ from the amplitude $A_{S}(k)$ (47)) directly (see Fig. 9) and calculate

$$
n_{S}(k)=\frac{\ln A_{S}(k)}{\ln k}+1 .
$$



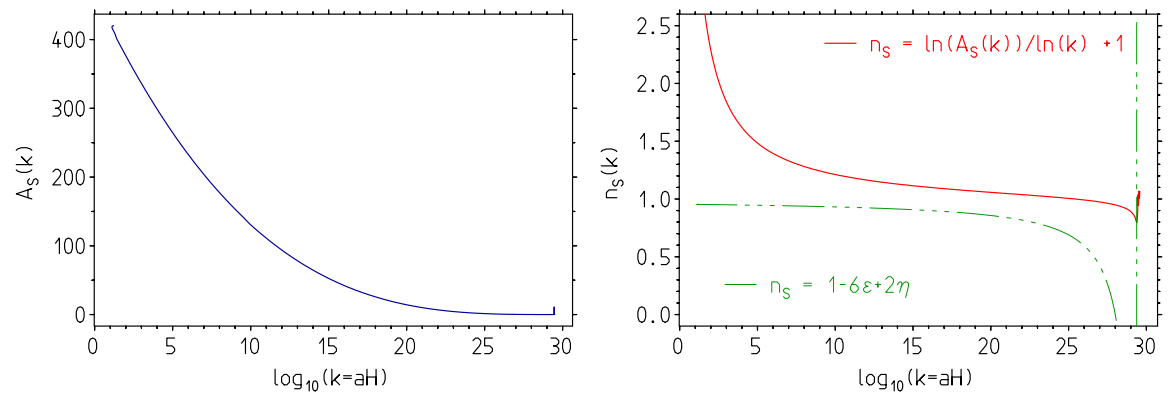

Figure 9: The spectral amplitude $A_{S}(k)$ as a function of $k=a H$ [left] and a comparison of the indices $n_{S}=\ln A_{S}(k) / \ln k+1$ versus $n_{S}=1-6 \varepsilon+2 \eta$ [right].
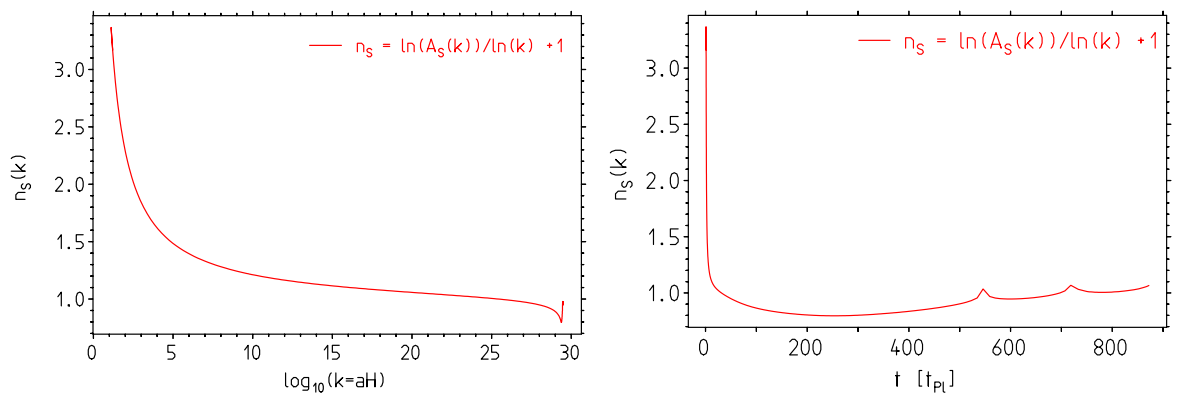

Figure 10: The index $n_{S}(k)$ extracted from $A_{S}(k)$ as a function of $k=a H$ [left] and as a function of time $t$ in units of $t_{\mathrm{Pl}}$.

We find the result displayed in Fig. 10 which differs substantially from the $n_{S}=1-6 \varepsilon+2 \eta$ as illustrated in Fig. 9 [right]. Actually $n_{S}(k)$ obtained via the exact definition provides results much closer to the reported observational value.

In our LEESM scenario the CMB temperature fluctuations would be directly related to the Higgs fluctuation field and CMB data would be a first direct access to measure the Higgs wave function in the early universe. With the above estimates we obtain $n_{S} \approx 1.067$ for $t \approx 870 t_{\mathrm{Pl}}$ and $n_{S} \approx 0.866$ at $t \approx 450 t_{\mathrm{Pl}}$, which corresponds to the end of slow-roll inflation. These results confront with the recent Planck mission result [9] $n_{S}=0.9603 \pm 0.0073$. I have not yet estimated the uncertainty, which however is expected to be large enough not to be in plain conflict with the data. More importantly, as we learn form Fig. 10, $n_{S}$ extracted via (48) is moderately below unity before it reaches values slightly above unity when we approach $\mu_{0}$. In comparing "theory with experiment" we should be aware that the observed spectrum is extracted over a cut $k$-range in the tail $k_{\min }<k$ of the amplitude $A_{S}(k)$ where the signal is not buried in the noise, which means $k<k_{\max }$. This leads to a much better agreement, but requires to take into account the cuts adopted in extracting the 
index from the CMB data.

The key lesson here is that, assuming $C(\mu)$ and $\lambda(\mu)$ being given, the spectral index is a monitor for the value of the otherwise unknown Higgs field $\phi(\mu)$, where $\mu$ is the scale accessible to observation. Once we kow $\phi(\mu)$ at some $\mu$ we can determine $\phi\left(M_{\mathrm{Pl}}\right)$ at the Planck scale, by solving the evolution equations from $\mu$ to $M_{\mathrm{Pl}}$.

Our analysis is a first estimate of the gross features of this Higgs inflation scenario. Here the SM Higgs field (actually four real heavy scalar fields of equal mass) is the inflaton scalar field, in the symmetric phase of the SM. In SM inflation the couplings of the inflaton to other particles are known, which is important for both the dynamics of inflation and reheating. As inflation pushes the system out of equilibrium through dramatic cooling, the effective couplings could change as much such that inflation gets stopped premature. The following reheating could reverse the process, such that the sign of the Higgs potential mass term could actually alternate for some short time interval. A detailed study of relaxation times of processes participating is required here. The question is whether at the Planck scale we have a system in thermal equilibrium. Then it could well be that inflation and the subsequent Higgs mechanism go so fast that the screening and antiscreening processes are slow enough such that an oscillatory inflation-reheating era is avoided. In our evaluation above we have assumed that the couplings follow as in thermal equilibrium and reheating has not yet been taken into account.

Concerning the running of couplings, relevant are the virtual processes which are dressing the top quark Yukawa coupling in $H t \bar{t}$ and the Higgs self-coupling in $H H \rightarrow H H, W W, Z Z$ as well as those relevant for the gauge couplings. At first sight a substantial change of the couplings between $\mu_{0}$ and $M_{\mathrm{Pl}}$ is not expected if we look at Table 1, however, this is not necessarily true as the $\beta$-functions utilized so far do not take into account that above $\mu_{0}$ the Higgses are very heavy. Above $\mu_{0}$ the four heavy Higgses $H, \phi, \phi^{ \pm}$do not contribute any more to the coefficients of the $\beta$-functions and the corresponding scalar loop contributions should be dropped. The corresponding changes have to be worked out yet. Besides the gauge boson self-couplings only the $H, \phi \rightarrow f \bar{f}$, the $\phi^{ \pm} \rightarrow f \bar{f}^{\prime}$ and the quartic $H H \rightarrow W W, Z Z, \phi^{+} \phi^{-} \rightarrow \gamma \gamma, \cdots$ type couplings are effective. The latter all include two heavy Higgs fields and two gauge boson fields and are barely effective in this phase. All $H \rightarrow W W$ single Higgs to two gauge bosons are absent in the symmetric phase (in the broken phase they are induced by the Higgs mechanism and proportional to the Higgs VEV $v$ ), such that they are not effective in Higgs decays. A detailed analysis is beyond the scope of the present work. One point is clear however, the seemingly minor differences in the running parameters between $\mu_{0}^{\prime}$ and $M_{\mathrm{Pl}}$ have a dramatic impact on the value of the coefficient $C^{\prime}(\mu)$ of the quadratic divergence, namely, the latter is zero at $\mu_{0}^{\prime}$, while it is magnified by the huge factor $M_{\mathrm{Pl}}^{2}$ away from $\mu_{0}^{\prime}$. It is responsible for the Higgs phase transition on the one hand as well as for the large Higgs masses in the symmetric phase above $\mu_{0}^{\prime}$ on the other hand. The running of the couplings in the symmetric phase thus has a dramatic impact on the specific properties of inflation. 


\section{Comment on reheating and baryogenesis}

The Higgses near the Planck scale have an effective mass about $m_{H 0} \simeq 3.6 \times$ $10^{17} \mathrm{GeV}$ and thus can be produces in processes like $W W \rightarrow H H$ or $t \bar{t} \rightarrow H$ at times before the phase transition takes place provided the temperature is above the corresponding Higgs mass thresholds $2 m_{H 0} \sim 8.4 \times 10^{30}{ }^{\circ} \mathrm{K}$ or $m_{H 0}$ $\sim 4.2 \times 10^{30}{ }^{\circ} \mathrm{K}$, respectively. This may not be too relevant as the heavy Higgses are primordial fields of the Planck medium. A Higgs there has a width dominated by $H \rightarrow t \bar{t}$ decay with

$$
\begin{aligned}
\Gamma_{H} & \simeq \Gamma(H \rightarrow t \bar{t}) \simeq \frac{m_{H 0}}{16 \pi} N_{c} y_{t}^{2}\left(M_{\mathrm{Pl}}\right) \\
& \simeq 7.35 \times 10^{-3} m_{H 0} \simeq 2.65 \times 10^{15} \mathrm{GeV}
\end{aligned}
$$

which yields a life time

$$
\tau_{H}=1 / \Gamma_{H} \simeq 2.5 \times 10^{-40} \mathrm{sec},
$$

which actually, for this process, supports the argument that, for some time, the effective couplings essentially do not change when the system is driven out of equilibrium. Here we used our result $y_{t}\left(M_{\mathrm{Pl}}\right)=0.3510$. We note that $\tau_{H}$ is large in terms of the Planck time $t_{\mathrm{Pl}} \simeq 5.4 \times 10^{-44}$ sec as well as the bare Higgs transition time $t_{H} \approx 4.7 \times 10^{-41} \mathrm{sec}$ and the effective Higgs transition time $t_{H}^{\prime} \approx 8.5 \times 10^{-40} \mathrm{sec}$. The drop of the $V(0)$ happens at $t_{\mathrm{CC}} \approx 2.1 \times 10^{-40} \mathrm{sec}$, halfway between $t_{H}$ and $t_{H}^{\prime}$.

The SM predicts that the Higgses produce top-anti-top quarks most abundantly. In addition we can say that $\Gamma_{H} \ll H(t)=\dot{a}(t) / a(t)$ as $H(t) \geq$ $7.2 \times 10^{16} \mathrm{GeV}$ during inflation, and actually until the drop of the CC discussed below. The heavy Higgses represent decaying non-relativistic matter such that their density scales with time as

$$
\rho_{\phi}(t)=\rho_{\phi}\left(t_{i}\right)\left(a_{i} / a(t)\right)^{3} \mathrm{e}^{-\Gamma_{H}\left(t-t_{i}\right)},
$$

as a solution of $\dot{\rho}_{\phi}+3 H \rho_{\phi}+\Gamma_{H} \rho_{\phi}=0$. The energy density of top/anti-top quarks produced by the Higgs decays satisfies the conservation equation (see e.g. Ref. [51])

$$
\dot{\rho}_{t}+3 H\left(\rho_{t}+p_{t}\right)=\Gamma_{H} \rho_{\phi}
$$

with $H^{2}=\ell^{2}\left(\rho_{\phi}+\rho_{t}+\cdots\right)$, and since the top quarks are relativistic (in the symmetric phase) $p_{t}=\rho_{t} / 3$ such that

$$
\begin{aligned}
\rho_{t} & =\Gamma_{H} \rho_{\phi}\left(t_{i}\right) a^{3}\left(t_{i}\right) / a^{4}(t) \int_{t_{i}}^{t} \mathrm{~d} t^{\prime} a\left(t^{\prime}\right) \mathrm{e}^{-\Gamma_{H}\left(t^{\prime}-t_{i}\right)} \\
& \leq \Gamma_{H} \rho_{\phi}\left(t_{i}\right) a^{3}\left(t_{i}\right) / a^{4}(t) \int_{t_{i}}^{t} \mathrm{~d} t^{\prime} a\left(t^{\prime}\right)
\end{aligned}
$$


At these times the energy density is still dominated by the inflaton, such that $a(t)=a\left(t_{i}\right)\left(t / t_{i}\right)^{2 / 3}$ and hence [51]

$$
\rho_{t}=\frac{3}{5} t_{i} \Gamma_{H} \rho_{\phi}\left(t_{i}\right)\left(\frac{t_{i}}{t}\right)^{8 / 3}\left(\left(\frac{t}{t_{i}}\right)^{5 / 3}-1\right) .
$$

The maximum is reached for $t=(8 / 3)^{3 / 5} t_{i}$ with $H\left(t_{i}\right)=2 / 3 t_{i}$, still well consistent with the assumption $\Gamma_{H} \ll H\left(t_{i}\right)$. The maximum matter density is then constrained by the bound

$$
\begin{aligned}
\rho_{t \max } & \leq(3 / 8)^{8 / 5} t_{i} \Gamma_{H} \rho_{\phi}\left(t_{i}\right)=0.139\left(\Gamma_{H} / H\left(t_{i}\right)\right) \rho_{\phi}\left(t_{i}\right) \\
& \simeq 0.139 \frac{3 \sqrt{3} y_{t}^{2}\left(M_{\mathrm{Pl}}\right)}{64 \pi \sqrt{\pi}} M_{\mathrm{Pl}}^{3} m_{H 0} \simeq 1.6 \times 10^{71} \mathrm{GeV}^{4} .
\end{aligned}
$$

In fact, the numerical estimate for the true maximum yields $\rho_{t \max } \simeq 1.2 \times$ $10^{71} \mathrm{GeV}^{4}$ reached at $t \simeq 1.74 t_{\mathrm{Pl}}$, well within the estimated bound. The main difference is due to taking properly into account the Higgs width. The evolution of $\rho_{t}$ is also displayed in Fig. 3 These values compare to the cosmological constant vacuum density

$$
\begin{aligned}
\rho_{\phi} & =V(0)+\frac{m^{2}\left(\mu_{0}\right)}{2} \phi_{e}^{2}+\frac{\lambda\left(\mu_{0}\right)}{24} \phi_{e}^{4} \approx V(0) \\
& \simeq 3.5 \times 10^{-6} M_{\mathrm{Pl}}^{4} \simeq 7.67 \times 10^{70} \mathrm{GeV}^{4} .
\end{aligned}
$$

After the produced $t \bar{t}$ pairs have been interacting sufficiently often to thermalize, the top flavored medium has a temperature $\rho_{t}=g_{*} \pi^{2} T^{4} / 30$ and the maximum reheating temperature reaches

$$
T_{\max }=\left(\rho_{t \max } /\left(g_{*} \pi^{2} / 30\right)\right)^{1 / 4} \simeq 8.9 \times 10^{30}{ }^{\circ} \mathrm{K},
$$

with $g_{*}=12 \frac{7}{8}$ for top quarks. As reheating temperature one defines $[52]$

$$
\begin{aligned}
T_{\mathrm{RH}} & \equiv T\left(t=\tau_{H}\right) \simeq 0.55 g_{*}{ }^{-1 / 4}\left(\Gamma_{H} M_{\mathrm{Pl}}\right)^{1 / 2} \\
& \approx 1.5 \times 10^{-2} M_{\mathrm{Pl}} \simeq 2.1 \times 10^{30}{ }^{\circ} \mathrm{K},
\end{aligned}
$$

usually considered to be the begin of the radiation dominated phase of the universe.

The physical "charged" channels $H^{+} \rightarrow t \bar{b}$ and $H^{-} \rightarrow b \bar{t}$ have by a factor $y_{b} / y_{t}$ reduced rates and $H \rightarrow b \bar{b}$ is reduced by $\left(y_{b} / y_{t}\right)^{2}$. Very important are the "charged" decays $H^{+} \rightarrow t \bar{d}, u \bar{b}$ and $H^{-} \rightarrow d \bar{t}, b \bar{u}$ which exhibit the dominant complex CP-violating CKM [53] matrix-element:44. Also the subsequent

\footnotetext{
${ }^{4}$ Present data [6] yield $V_{t d} \sim A \lambda^{3}(1-\rho-\mathrm{i} \eta)$ and $V_{u b} \sim A \lambda^{3}(\rho-\mathrm{i} \eta)$ with $\lambda=0.22535 \pm$ $0.00065, A=0.811_{-0.012}^{+0.022}, \rho=0.131_{-0.013}^{+0.026}$ and the CP violating phase $\eta=0.345_{-0.014}^{+0.013}$. The overall $\mathrm{CP}$ violation is characterized by the Jarlskog invariant $J=\lambda^{6} A^{2} \eta \approx 2.97 \times 10^{-5}$ and the Jarlskog determinant $\delta_{J}=J\left(y_{t}^{2}-y_{c}^{2}\right)\left(y_{t}^{2}-y_{u}^{2}\right)\left(y_{c}^{2}-y_{u}^{2}\right)\left(y_{b}^{2}-y_{s}^{2}\right)\left(y_{b}^{2}-y_{d}^{2}\right)\left(y_{s}^{2}-y_{d}^{2}\right) \approx$ $9.94 \times 10^{-24}$ a tiny number, which however together with a large Higgs induced dark energy term, decaying predominantly into heavy flavors, may lead to realistic magnitude for the baryon-asymmetry.
} 
processes $t \rightarrow W d$ or $b \rightarrow W u$ exhibit the same CKM couplings, which are able to contribute to the baryon-asymmetry.

In standard baryogenesis scenarios some unknown heavy particle $X$, usually assumed to have been pair-created in the hot early universe, when $k_{B} T$ exceeded all particle masses, are assumed to decay into pairs of particles of different baryon and lepton number. This can produce a baryon- or lepton-asymmetry, respectively, if $C$ and $C P$ are violated as they are in the SM. The necessary $B$ violation are assumed to be produced by appropriate dimension 6 four-fermion operators [54 56]. In our LEESM scenario, the latter are naturally expected as $\left(E / \Lambda_{\mathrm{Pl}}\right)^{2}$ terms in the low energy expansion. At the scale of the EW phase transition the Planck suppression factor is $1.3 \times 10^{-6}$. The basic asymmetry parameter is $\epsilon=B_{X}+B_{\bar{X}}=r-\bar{r}$, where $B$ are the baryon numbers produced and $r$ and $\bar{r}$ are branching fractions, which would be equal $(\bar{r}=r)$ if $C$ and/or $C P$ would be conserved [52]. One assumes that the particle numbers in thermal equilibrium agree $n_{X}=n_{\bar{X}} \sim n_{\gamma}$ and the baryon number density in units of the entropy density is given by $n_{B} / s \sim \epsilon n_{X} / g_{*} n_{\gamma} \sim \epsilon / g_{*}$. Here $g_{*}$ is the number of relativistic degrees of freedom produced in the decays. Typical values are $\epsilon \sim 10^{-8}$, sufficient to reproduce the observed baryon asymmetry $\eta_{B} \sim 10^{-10}$.

When $k_{B} T(t)>M_{X}$ and $H(t)<\Gamma_{X}, X$-creation and $X$-annihilation are equally efficient and the expansion rate is slow enough such that there is sufficient time for the system to stay in thermal equilibrium. No net asymmetry can develop in this case. During inflation, until the drop of the CC at $t_{\mathrm{CC}} \approx$ $2.1 \times 10^{-40} \mathrm{sec}$, we actually have a large Hubble constant $H=\dot{a} / a(t) \gg \Gamma_{X}$. In this case, as long as $k_{B} T(t)>M_{X}$ still $X$ production is effective and the various radiative components still follow the equilibrium distributions with rescaled temperature $T(t) \propto 1 / a(t)$ and the system behaves as if in thermal equilibrium. So, what we need is $H=\dot{a} / a(t) \gtrsim \Gamma_{X}$ and $k_{B} T(t)<M_{X}$ in which case the inverse decay is blocked and the system is truly out of equilibrium and the out-of-equilibrium condition for baryogenesis is satisfied. Our scenario is somewhat different. Our $X$ particle are the four very heavy primordial Higgses from the Planck medium, the properties of which we know, in particular their masses, widths and branching fractions, as well as their $C$ and $C P$ violating coupling structure. The system seems to be out of equilibrium way down to the Higgs transition point $\mu_{0}^{\prime}$, and the immediately following EW phase transition, which is of pronounced 1st order type triggered by the sing change in the effective mass $m^{\prime 2}$. In our case, Higgs reproduction gets stopped somewhat earlier by the drop of the CC (see Fig. 14). The Higgs width there is about $\Gamma_{H} \approx 1.39 \times 10^{-4} M_{\mathrm{Pl}}$. Before, the drop of the CC at time $t \approx 3796 t_{\mathrm{Pl}}$ the Hubble constant is $H \approx 2.03 \times 10^{-4} M_{\mathrm{Pl}}$, while after the drop, at $t \approx 3810 t_{\mathrm{Pl}}$, $H$ has decreased suddenly to $H \approx 5.50 \times 10^{-12} M_{\mathrm{Pl}}$ and Higgs recreation is stopped. At this stage the inflaton field is converted completely into radiation. A detailed analysis and numerical estimate of the baryon asymmetry which one can obtain in this way is missing at this point. If we adopt the analysis elaborated in Ref. 52 as the way-out-of-equilibrium scenario, which covers our Higgs inflation scenario, the baryon asymmetry is given by $n_{B} / s \simeq \epsilon T_{\mathrm{RH}} / m_{\phi}$, with reheating temperature $T_{\mathrm{RH}}$ given in Eq. (59)). Then, assuming $\epsilon \sim 10^{-8}$ one 
estimates $n_{B} / s \simeq 5.0 \times 10^{-9}$. What remains to be worked out is the basic $B$ violation parameter $\epsilon$. The value adopted looks very plausible in our scenario but depends on unknown couplings accompanying the $B$ number violating dimension 6 operators.

It is interesting to see that the large cosmological constant energy density is converted predominantly into, yet massless, top flavored matter/antimatter, which is known to undergo matter-antimatter annihilation. It seems that we are all descendants from a dense top-anti-top sea, which cascades down to the light quark world we live in. Thereby we have to undergo all CKM physics. During the EW phase transition the particles acquire their mass and heavier particles start to decay into lighter ones. The effective mass hierarchy mix-up, illustrated by Fig. 2 of Ref. 7, likely plays a role here. Close to the phase transition the effective top-quark mass is not yet clearly heavier than the effective $W$-boson mass, for example. So top quarks could be quasi-stable and form toponium states for some time after the EW phase transition has taken place, and before they decay via $t \rightarrow W b$ and then cascade down to the light quarks. It is interesting to note that in the symmetric phase those fermion modes win which have the strongest Yukawa coupling, i.e. the top quark flavor. After the EW phase transition, because of the mass-coupling relation, when the universe cools further down, the heavier particles decay into lighter ones. Now the modes with the weakest Yukawa couplings win, as they have larger phase space, and survive in form of normal matter. This also may shed some light on the question about the huge mass hierarchy between the heaviest and the lightest quark $M_{t} / m_{u} \sim 10^{5}$, which in our scenario must be large enough to get a sufficient amount of normal light matter. Whether this intricate EW phase transition scenario leaves room for dark matter relicts is an open problem.

It is important also to remind us that it is the EW phase transition 5 which puts the SM into operation with all its properties we are familiar with. QED, in particular, with its special role in the development of structure during the following evolution of the universe comes into existence only with the EW phase transition. Normal photon radiation, the photon as the only massless particle, particles of definite charge, matter-antimatter annihilation into light, Dirac fields and all that show up in the broken phase only. Particularly interesting is what happens with the most abundantly produced top quarks, which decay into the lighter flavors. It is interesting that this proceeds through the $b$-quark sector, which exhibits the large component of CKM CP-violating phase. Since, in our scenario, the EW phase transition is carried along by the Higgs transition, the system likely would intermittently be far from equilibrium. Both, are key ingredient for the explanation of the baryon-asymmetry and for understanding baryogenesis. So, possibly, in this scenario the origin of the baryon-asymmetry could well have its explanation within the SM.

One of the main consequences of our LEESM scenario is that the SM hierarchy problem is not a problem of the SM but the solution for inflation and

\footnotetext{
${ }^{5}$ Because of the finite temperature effect the EW phase transition takes place always after the Higgs mechanism.
} 
dark energy in the early universe (see Ref. 8 for more details). Since the Higgs VEV $v\left(\mu^{2}\right)$ emerges as a low energy phenomenon (order parameter) from the phase transition regime at or near $\mu_{0}^{\prime}$, where the quadratic divergence is nullified, there is no hierarchy problem in the broken phase since as a result of the well known mass coupling relations all masses, including the Higgs mass itself, have values proportional to the EW scale $v$, up to factors essentially given by the SM couplings, which in any case depend logarithmically on the scale only.

\section{The cosmological constant}

The cosmological constant problem (see e.g. Ref. 57]) has been a persisting problem for a long time already. It usually is considered to be the most severe fine tuning problem within the $\mathrm{SM} 6$. As we have seen, the SM predicts a huge time-dependent $\mathrm{CC}$, at $M_{\mathrm{Pl}}$ equivalent to $\rho_{\phi} \simeq V(\phi) \sim 2.77 M_{\mathrm{Pl}}^{4} \sim 6.13 \times$ $10^{76} \mathrm{GeV}^{4}$, for the given initial field value (28), while the value observed today is $\rho_{\text {vac }}=\mu_{\Lambda}^{4}$ with $\mu_{\Lambda} \sim 0.002 \mathrm{eV}$ ! In the unbroken phase the CC is essentially provided by the quartically enhanced Higgs potential $V(\phi)$, which identifies the $\mathrm{CC}$ as a field, however, with a weakly scale dependent vacuum contribution $V(0)$, which shortly after the begin of inflation starts to be dominating. As we already know, later, the Higgs mechanism contributes $-\frac{\lambda}{24} v^{4} \sim 1 \times 10^{9} \mathrm{GeV}^{4}$ to $V(0)$ via the non-vanishing VEV $v$. Although much smaller than $V(0)$ at scales $\mu>\mu_{\mathrm{CC}}$, it represents a large negative contribution to the vacuum energy density 7. Much later, after the universe has cooled down to a temparature of about $150 \mathrm{MeV}$, QCD undergoes the chiral phase transition which yields another substantial contributions to the vacuum density 8 . This shows that in any case

\footnotetext{
${ }^{6}$ As emphasized in Ref. 58 [59, one should note that in condensed matter systems the macroscopic ground state energy density is not determined by the quantum fluctuations but rather by temperature and pressure of the system which are determined by the environment which can change with time.

${ }^{7}$ At scale $\mu_{0} \sim 1.4 \times 10^{16}$ we have $\lambda \sim 0.1393$ and $v \sim 638 \mathrm{GeV}$ such that $\frac{\lambda}{24} v^{4} \sim$ $9.6 \times 10^{8} \mathrm{GeV}^{4}$. Converted with the factor $\kappa=8 \pi G$, it corresponds to a shift $\Delta \Lambda_{\mathrm{EW}}=$ $\kappa \Delta \rho_{\mathrm{vac}} \simeq-0.44 \mathrm{~cm}^{-2}$ in the cosmological constant $\Lambda$, while the observed value is given by $\Lambda_{\text {obs }}=\kappa \rho_{\text {crit }} \Omega_{\Lambda}=1.6517 \times 10^{-56} \mathrm{~cm}^{-2} . \Omega_{\Lambda}=0.67_{-0.023}^{+0.027}$ is the dark energy fraction of the critical energy density $\rho_{\text {crit }}=3 H_{0}^{2} \kappa^{-1}=1.878 \times 10^{-29} h^{2} \mathrm{gr} / \mathrm{cm}^{3}$ with $h=0.67 \pm 0.02$ for which the universe is flat 60 .

${ }^{8}$ The chiral phase transition of QCD which leads to quark condensates contributing

$$
T_{\mu \nu \mathrm{QCD}}^{\mathrm{vac}}=-\left\langle 0\left|\mathcal{L}_{\mathrm{QCD}}\right| 0\right\rangle g_{\mu \nu}=\left\{m_{u} \bar{u} u+m_{d} \bar{d} d+m_{s} \bar{s} s+\cdots\right\} g_{\mu \nu}
$$

to the cosmological constant, has to be reconsidered under the aspect that the relation between bare an renormalized quantities are physical in the low energy effective approach. Maybe it is possible to give a more precise meaning also to the gluon condensate within this context. The quark condensates yield $\rho_{q q}^{\mathrm{vac}}=m_{u} \bar{u} u+m_{d} \bar{d} d+m_{s} \bar{s} s+\cdots \simeq-2 \times 0.098 \mathrm{GeV}^{4}-0.218 \mathrm{GeV}^{4}$ or

$$
\Lambda_{q \bar{q}} \simeq-1.0542 \times 10^{-12} \mathrm{~cm}^{-2} .
$$

The gluon condensate is not well defined. A typical value found in the literature is $\left\langle\frac{\alpha_{s}}{\pi} G G\right\rangle \sim$ $(0.389 \mathrm{GeV})^{4}$ which would overcompensate the negative quark condensate terms and would change the result to

$$
\Lambda_{\mathrm{QCD}} \simeq 1.668 \times 10^{-10} \mathrm{~cm}^{-2} .
$$
}


the cosmological constant, represented by the dark energy density, obviously is changing during the evolution of the universe. As discussed earlier, the decay of the Higgs inflaton transforms the cosmological constant, first into top radiation, which gets reduces by $a(t)^{-4}$ until the EW phase transition, and at the EW phase transition into matter which afterwards diminishes like $a(t)^{-3}$ to date. Thus the cosmological constant [scaling with $a(t)^{0}$ ] could have been decimated on account of other energy forms which get largely diluted by the expansion of the universe. It is clear that the total energy density as a function of time

$$
\rho(t)=\rho_{0, \text { crit }}\left\{\Omega_{\Lambda}+\Omega_{0, \mathrm{k}}\left(a_{0} / a(t)\right)^{2}+\Omega_{0, \text { mat }}\left(a_{0} / a(t)\right)^{3}+\Omega_{0, \mathrm{rad}}\left(a_{0} / a(t)\right)^{4}\right\}
$$

only reflects a present-day snapshot. The $\Omega$ 's representing the present fraction of dark energy, curvature, matter and radiation of the total universal Einsteinde Sitter density $\rho_{0, \text { crit }}$ (globally flat space), do not really describe the evolution of the energy density in the history of the universe, because physical processes transform different forms of the energy. Such transmutations in most cases are well understood and of course have been well accounted for most of the known processes. However, to my knowledge, it does not take into account the scenario we advocate, namely that the universe has undergone the Higgs mechanism after inflation. In other words, the cosmological "fine tuning" problem could turn out to be a pseudo problem as the dynamics, subject to energy balance constraints, resolves itself, in the sense that the cosmological constant is decaying into other forms of radiation and matter which naturally decrease with time. The main effect, however, is the vacuum rearrangement during the Higgs transition as we a going to argue now.

We know that for the early cosmological evolution the CC plays a key role. The Higgs potential and the Higgs field dynamics are all-dominant shortly after the big bang and until the Higgs mechanism is ignited by the sign-flip in our key running function $C(\mu)$ Eq. (2) or $C^{\prime}(\mu)$ Eq. (39). As we have seen, the Higgs fluctuation field during inflation decays exponentially and eventually is not able to yield the required blow-up exponent $N_{e}$. In fact the predictable constant $V(0)$ Eq. (37), provided by quartically "divergent" Higgs loops, supplies the necessary persisting blowing-up of the universe. This represents the intrinsic CC which depends on $m^{2}(\mu)$ and $\lambda(\mu)$ but persists to be large until the bare Higgs mass term changes sign and the vacuum reorganizes itself (see Fig. 11). From

$$
V(\phi)=V(0)+\frac{m^{\prime 2}}{2} \phi^{2}+\frac{\lambda}{24} \phi^{4} \quad \text { with } m^{\prime 2}<0,
$$

we have a minimum at $\phi_{0}^{2}=-\frac{6 m^{\prime 2}}{\lambda}$ and an effective bare Higgs mass $m_{H}^{2}=$ $-2 m^{\prime 2}$. At the minimum $V\left(\phi_{0}\right)=V(0)+\Delta V\left(\phi_{0}\right)$ with $\Delta V\left(\phi_{0}\right)=\frac{m^{\prime 2}}{2} \phi_{0}^{2}+$ 


$$
\begin{aligned}
\frac{\lambda}{24} \phi_{0}^{4}=-\frac{3}{2} \frac{m^{\prime 4}}{\lambda}, & \text { and using }{m^{\prime}}^{2}=\frac{\mu_{m}+C+\lambda}{2} \Xi \text { with } \Xi=\frac{M_{\mathrm{Pl}}^{2}}{16 \pi^{2}}, \text { we find } \\
V_{\min }= & V(0)+\Delta V\left(\phi_{0}\right)=\frac{m_{0}^{2}}{2} \Xi+\frac{\lambda}{8} \Xi^{2}-\frac{3}{2} \frac{m^{\prime}}{\lambda} \\
= & \frac{1}{8} \frac{M_{\mathrm{Pl}}^{4}}{\left(16 \pi^{2}\right)^{2}}\left(2\left(\mu_{m}+C(\mu)+\lambda(\mu)\right)-\lambda(\mu)\right. \\
& \left.-\Theta\left(-\left(\mu_{m}+C(\mu)+\lambda(\mu)\right)\right) \frac{3}{\lambda(\mu)}\left(\mu_{m}+C(\mu)+\lambda(\mu)\right)^{2}\right) .
\end{aligned}
$$

Here $\Theta(x)$ denotes the step function: $\Theta(x)=1, x>0$ and $=0, x<0$. The subtraction of the "jump" $\Delta V\left(\phi_{0}\right)$ applies as soon as ${m^{\prime}}^{2}<0$, equivalently, $\mu_{m}+C(\mu)+\lambda(\mu)<0$, which is in the Higgs phase. Here again we observe an intriguing structure, which exhibits a zero not far away from the zero of $C(\mu)$ as we have $\lambda>0$ but small and $C(\mu)$ is growing negative below its zero. So actually, the effective $\mathrm{CC}$ counterterm has a zero, which again is a point where renormalized and bare quantities are in agreement:

$$
\rho_{\Lambda 0}=\rho_{\Lambda}+\frac{M_{\mathrm{Pl}}^{4}}{\left(16 \pi^{2}\right)^{2}} X(\mu)
$$

with $X(\mu)=0$ close to the zero of $C(\mu)$. The impact on the various terms in the potential is displayed in Fig. 14. Interestingly, the functions $C(\mu)$ and $X(\mu)$, which nullify the difference between renormalized and bare mass and vacuum density, respectively, are strongly correlated, implying that the corresponding zeros are effective at comparable scales. It means that short range and long range regime match in a vicinity of the Higgs transition spot. One thing is clear, there is no dramatic fine tuning problem as anticipated usually. The mechanisms both for the quadratic- as well as for the quartic-enhancements are not a matter of taking differences between two independent huge numbers, but a matter of a huge number which is multiplied by a function exhibiting a zero by cancellation of normal sized effective couplings in our cases. That is how selforganized fine-tuning works. What is also interesting is that these mechanisms are possible only by cancellations between bosonic and fermionic contributions. In Fig. 12 we show the location and structure of the correlated zeros. The sign-change for the bare mass $m_{0}^{2}=m^{2}+\delta m^{2}$ occurs at the zero of $\mu_{m}+C(\mu)$, where $\mu_{m}$ accounts for a small positive possible contribution from a renormalized mass (in Plank mass units). In fact, because of the necessary Wick reordering to account for the vacuum contributions the true effective mass is $m_{0}^{2}+\frac{\lambda}{2} \Xi$ such that the effective Higgs transition is ruled by $\mu_{m}+C(\mu)+\lambda(\mu)$ not by $\mu_{m}+C(\mu)$, as we see in Fig. 12 this shifts the location of the Higgs transition to a lower value $\mu_{0}^{\prime} \simeq 7.7 \times 10^{14} \mathrm{GeV}$ ! Again, we point out that such results sensitively depend on the $\overline{\mathrm{MS}}$ input parameter values, and are expected to change if more precise parameter values and a better understanding (including higher order effects) of the matching relations have been achieved. Above the zero of $m^{\prime 2}$ the coefficient of the "amplifier" $\frac{M_{\mathrm{P} 1}^{4}}{\left(16 \pi^{2}\right)^{2}}$ is $2\left(\mu_{m}+C(\mu)+\lambda(\mu)\right)-\lambda(\mu)>0$, which induces a huge CC. As $\mu_{m}+C(\mu)+\lambda(\mu)$ flips sign we get a negative jump 


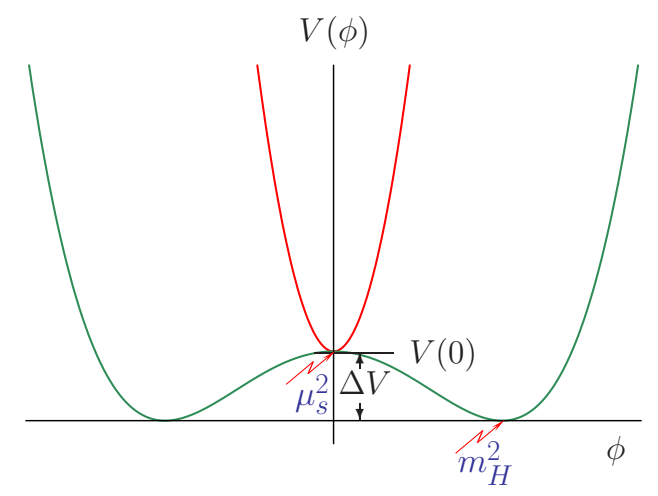

Figure 11: Vacuum rearrangement of the Higgs potential. At the zero of $X(\mu)$ of Eq. (63) $\Delta V=-V(0)$ nullifying the difference between bare and renormalized CC. The bare mass $m_{0}^{2}=\mu_{s}^{2}$ in the symmetric phase is correlated to the renormalized $m_{H}^{2}$ in the Higgs phase, as follows from the text.

$-\frac{3}{2} \frac{\left(\mu_{m}+C(\mu)+\lambda(\mu)\right)^{2}}{\lambda(\mu)}$. Surprisingly, at the zero of ${m^{\prime}}^{2}$ the quartic coefficient $X(\mu)=-\lambda$, i.e. $X(\mu)$ changes sign slightly before the Higgs transition at $\mu_{\mathrm{CC}} \simeq 5.01 \times 10^{15} \mathrm{GeV}$ ! Thus, again it is the running of the couplings and not the jump from the vacuum rearrangement which is responsible for attaining the zero, which is the matching point between bare and renormalized quantities!

In order to understand better what I mean by "self-organized fine-tuning" let us consider the lattice version of the SM. This is an obvious candidate for our Planck medium in the right universality class by construction and it is a non-perturbatively well-defied system. If we investigate this lattice SM at short distances we see the lattice structure as the true short distance world and we would see it to be in the symmetric phase and a relation like (11) or (63) to have a true physical meaning. In this short distance regime the bare system is the physical one as we have been anticipating in our description of the inflation era. In contrast, if we investigate its long range properties by probing appropriate observable quantities, we would see the effective renormalizable continuum field theory and the symmetry to be spontaneously broken. This requires parameters of the lattice system to be in a range which allows that long range order can be effective. As elaborated in Sect. 2 of Ref. [7, before we renormalize the long range correlations emerging from the lattice system, these indeed exhibit a residual dependence on the cutoff. However, the cutoff at long distances functions as a renormalization reference scale only and has lost its meaning as a cutoff9! This means that the cutoff can be renormalized away in favor of the $\overline{\mathrm{MS}}$ parameter $\mu$, for example. By low energy instruments we will no longer be able to probe the short distance structure, therefore we will not encounter any cutoff related effect and everything plays in the framework of the

\footnotetext{
${ }^{9}$ Formal criterion is that the RG in the cutoff $\Lambda$ takes a homogeneous form.
} 

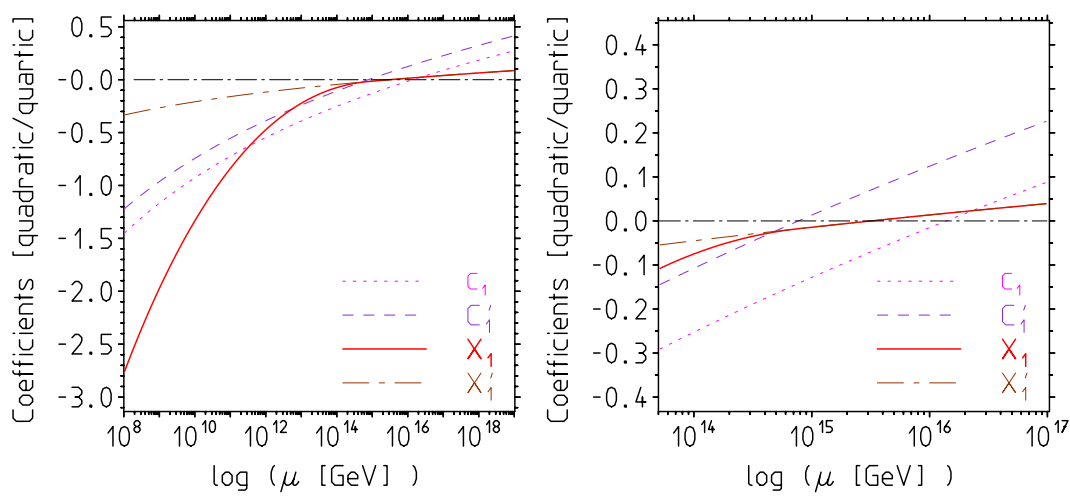

Figure 12: The correlated zeros of $C_{1}(\mu)$ in the quadratic and of $X_{1}(\mu)$ in the quartic Planck cutoff enhanced terms, respectively. Relevant are the effective coefficients $C_{1}^{\prime}=C_{1}+\lambda$ and $X_{1}$. Here $X_{1}^{\prime}$ represents the quartic coefficient not including the vacuum rearrangement jump, which does not actually affect the location of the zero.

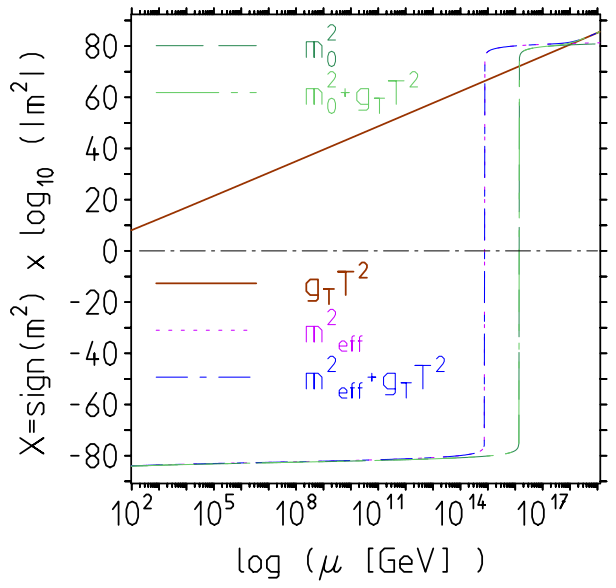

Figure 13: The effective mass vs. the bare mass including finite temperature effects. The latter have little effect on the location of the EW phase transition. The shift $C_{1} \rightarrow C_{1}^{\prime}=C_{1}+\lambda$ has a substantial effect on the scale of the Higgs transition. 


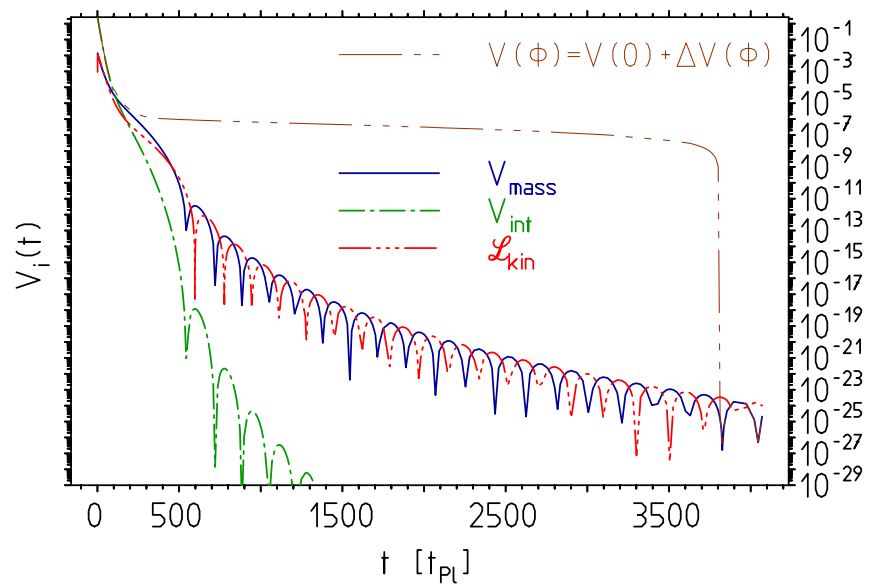

Figure 14: Here we show the same quantities as in Fig. 6 in an extended range exhibiting the vacuum rearrangement at $\mu_{\mathrm{CC}}$. After inflation the scene is characterized by a free damped harmonic oscillator behavior. Relevant scales are $\mu_{0} \simeq 1.4 \times 10^{16} \Leftrightarrow t \simeq 870 M_{\mathrm{Pl}}^{-1}$ at the zero of $m_{0}^{2}-m^{2}=0, \mu_{\mathrm{CC}} \simeq 3.1 \times 10^{15} \Leftrightarrow$ $t \simeq 4000 M_{\mathrm{Pl}}^{-1}$ where $\rho_{\Lambda 0}=\rho_{\Lambda}$ and $\mu_{0}^{\prime} \simeq 7.7 \times 10^{14} \Leftrightarrow t \simeq 15844 M_{\mathrm{Pl}}^{-1}$ the true Higgs transition point $m^{\prime 2}=0$.

renormalized low energy effective theory. But, how do we get ride of the cutoff in the mass renormalization? Indeed we have to tune the bare mass of the short distance system to criticality, i.e., tune $m_{0}^{2} \rightarrow m_{0 \text { crit }}^{2}$ such that $m^{2}=+0$. The renormalized mass now given by $m^{2}=m_{0}^{2}-m_{0 \text { crit }}^{2}$ then can be tuned to have any value we want. This is fine tuning par excellence, at least in the symmetric phase. However, this argument does not answer the question when we are in the spontaneously broken phase where long range order is taking over. I think that the hot Planck medium is exhibiting a plethora of modes where some conspiring ones are able to reach to long distances, exhibiting the masses we see. Why the minimum of the Higgs potential should not naturally be close to the one at zero of the symmetric phase? Why should it jump from zero to $M_{\mathrm{Pl}}$ suddenly? And if the location of the minimum $\phi_{0} \ll M_{\mathrm{Pl}}$ is natural, why $m_{H} \ll M_{\mathrm{Pl}}$ is not? Our calculation presented above proves that as the universe expands the spontaneous symmetry breaking phase develops continuously from the symmetric phase, i.e. as $m^{\prime 2}$ passes a zero $\phi_{0}^{2}(\mu)=-\frac{6 m^{\prime 2}}{\lambda}(\mu)$ moves smoothly from zero to whatever value, and in any case at some point matches its renormalized value. No low energy experiment is able to substantiate a supposed fine-tuning problem, and doing short distance experiments would probe the symmetric phase where a fine-tuning problem is not known to exist, at least as long as we do not know what the value of the renormalized mass in the symmetric phase is. 
The dynamical part now is the broken phase Higgs Lagrangian

$$
\mathcal{L}_{\text {Higgs }}=\frac{1}{2} \dot{\phi}^{2}+\frac{m_{H}^{2}}{2} \phi^{2}+\frac{\sqrt{3 \lambda} m_{H}}{6} \phi^{3}+\frac{\lambda}{24} \phi^{4} .
$$

where $m_{H}=m_{H}(\mu)$ is the renormalized $\overline{\mathrm{MS}}$ mass. The CC is also to be identified with some renormalized value, which we know must be small. The field equation (19) now reads

$$
\ddot{\phi}+3 H \dot{\phi}=-\left(m_{H}^{2} \phi+\frac{\sqrt{3 \lambda} m_{H}}{2} \phi^{2}+\frac{\lambda}{6} \phi^{3}\right) .
$$

I expect that the observed value of dark energy has to be considered as a phenomenological constraint. The reason is that $\rho_{\Lambda}$ is dependent on the Higgs field magnitude, which is not fixed by other observations, except maybe by CMB inflation data. In addition we have to keep in mind that our scenario is very sensitive to our basic parameters $C(\mu)$ and $\lambda(\mu)$, which were obtained by evolving coupling parameters over 16 orders of magnitude in scale. This cries for high precision physics to really settle the issue. Note however, that given the SM couplings everything here is a SM prediction without any extra assumption. In any case, what we learn is that the quartic divergences in the vacuum energy are not the problem, rather they are the solution providing inflation as a necessary consequence of the SM.

What about the other vacuum condensate contributions we expect to show up in the broken phase of the SM? First the Higgs transition contribution associated with a non-vanishing $\langle H\rangle=v$ now is accounted for by the "jump" $\Delta V\left(\phi_{0}\right)$ contributing to Eq. (62) and thus has been accounted for in the relation (63). What concerns the QCD condensates contribution mentioned earlier, this has to be reconsidered in our context of the LEESM. Presently, not even the sign of $\rho_{\mathrm{QCD}}^{\mathrm{vac}}$ is known for sure, so there is a chance that also this problem will find its solution.

\section{Conclusions}

The recent discovery of the Higgs at the LHC revealed a Higgs mass in a window which strengthens our believe into a low energy effective SM scenario, with a largely unknown medium at the Planck scale exhibiting the Planck mass as a cutoff. This may represents a dramatic change of the predominating paradigm concerning the "Path to physics at the Planck scale" which is the SUSY, GUT and strings driven "the higher the energy the more symmetry" belief. The LEESM scenario supports strongly an emergent look at what we see at long distances. The SM is a naturally emergent low energy structure, the world as seen from far away [7].

The Higgs discovery, together with the fact than non-SM physics has not yet shown up at the LHC, may have a dramatic impact on particle theory and particle physics all-together. We have shown that, under the conditions that the 
SM vacuum remains stable up to the Planck scale and that the quadratically enhanced Higgs mass counterterm exhibits a zero not far below the Planck scale, likely the SM largely summarizes the all-driving laws of physics which govern the evolution of the universe from its birth and possibly for all future. This likely brings high precision physics and high precision SM calculations in the focus of future developments as a tool to learn more about early cosmology. We note that close-by non-SM low energy emergent new physics is naturally expected to exist. The origin of dark energy or the strong CP problem definitely may find their solution in new not yet fully uncovered low energy emergent physics [7]. Should vacuum stability in the SM fail all this could be completely different 6163 .

What we have shown is that a CMB data consistent inflation scenario is possible solely on the basis of SM physics, with the Higgs being the driver. The big difference in comparison to alternative inflation scenarios is the fact that we almost perfectly know the properties of the inflaton, such that we are able to get true predictions, results which are more than more or less direct consequences of more or less plausible assumptions.

We essentially are left with two quantities which we have to constrain by data extracted from the observed CMB fluctuation patterns: the renormalized mass in the symmetric phase of the SM, and more importantly, the magnitude of the classical Higgs field at the Planck scale. The renormalized mass square we assumed to be subleading at the Planck scale such that $m^{2} \ll m_{0}^{2}$ at $M_{\mathrm{Pl}}$. This seems to be well supported by CMB data. The second assumption derives from the need of sufficient inflation, required to solve the CMB horizon problem, in particular. We found that a $25 \%$ enhancement of a field strength $\phi$ which yields an energy density $\rho_{\phi} \simeq V(\phi) \simeq M_{\mathrm{Pl}}^{4}$ is sufficient to provide the necessary magnitude of exponential growth of the FRW-radius. We include the Higgs vacuum diagrams as predicted by the SM and which provides a very weakly decreasing moderately big cosmological constant contribution $V(0)$, which depends only on the running values of $\lambda(\mu)$ and $C(\mu)$, the coefficient function which determines the enhanced effective Higgs mass. What at first sight looks to be a severe cosmological constant problem, resolves itself, as the difference between the renormalized and the bare cosmological constant vanishes near slightly above the Higgs transition point by running of the SM couplings also in this case. At this point bare and renormalized values of the cosmological constant coincide and the renormalized value may be identified with the observed tiny dark energy term, which in spite of its smallness remains the dominant contribution of today's energy density in the universe, as we know. This does not exclude the possibility that a better understanding of the dynamics of the EW phase transition would allow us to predict $\rho_{\Lambda}$. Likely, also today's value of the dark energy is provided by the Higgs as the source which is continuously blowing energy into our universe providing the accelerated expansion. This corresponds to a tiny continuous inflation. Remarkably this does not contradict energy conservation as the cosmological constant is the only covariant quantity which is covariantly conserved by-itself.

This also sheds new light on the hierarchy problem, usually considered to be a fine-tuning problem in the sense that a relatively small physical quantity is the 
difference of two uncorrelated huge numbers. In fact the huge cutoff terms turn out to be multiplied by an $O(1)$ function which can exhibit a zero, which actually at some point removes the cutoff dependence and provides a spot at which the bare short distance world matches with our renormalized long distance world.

What is new here is that we have evidence that the disentanglement between short distance and long distance is complete. Quadratically as well as quartically blown-up quantities, natural in the bare system, decouple from low energy physics at the Higgs transition spot where long range order in form of the Higgs vacuum condensate $v$ sets the scale. Now long range effects shape what we are able to see. The large hierarchy $v / M_{\mathrm{Pl}}$ just tells us that our world is close below a second order phase transition point 10 . This is also what the theory of critical phenomena and emergent continuum field theory structures suggest.

One more remark should be made here: as I pointed out several times the spot in SM parameter space where SM inflation can work seems to be very narrow. The detailed SM inflation scenario, e.g. what are the predominant characteristics as a function of time in the evolution of the early universe, depends a lot on the precise value of the $\overline{\mathrm{MS}}$ top Yukawa coupling $y_{t}\left(M_{Z}\right)$ in particular. Thus details may change a lot when our knowledge of the parameters improve. Nevertheless, I think that this analysis raises hopes that at the end we will be able to establish the Higgs as the inflaton and as the supplier of dark energy.

\footnotetext{
${ }^{10} \mathrm{I}$ am referring here to the commonly known example of spontaneous magnetization in a ferromagnetic system: the magnetization $M$ is the order parameter (corresponding to our $v$ in the SM), the result of long range collective behavior of the spins of the system. Above a critical temperature $T_{c}$ there is no spontaneous magnetization $M(T) \equiv 0$ as $T>T_{c}$. Below $T_{c}$, as we lower $T, M(T)$ is a monotonically increasing function with its maximum value at $T=0$. If we approach $T_{c}$ from below $M(T)$ continuously decreases to zero at $T_{c}$, and hence can be arbitrarily small depending on how close we are to the critical point $(T, M)=\left(T_{c}, 0\right)$, which corresponds to a second order phase transition point, the end point of a continuous family of first order phase transitions corresponding to possible jumps in the magnetization $M(T) \leftrightarrow-M(T)$ when $T<T_{c}$.
} 


\section{Acknowledgments}

Many thanks to Paul Söding for critically reading the manuscript and his helpful comments and questions. I furthermore thank Oliver Bär, Nigel Glover, Mikhail Kalmykov and Daniel Wyler for their interest and for many clarifying discussions. I gratefully acknowledge the interest and patience of my wife Marianne, as many of the ideas worked out here came to my mind when I tried to explain her what I think the role the Higgs could have played in the evolution of the early universe.

\section{References}

[1] G. Aad et al. [ATLAS Collab.], Phys. Lett. B 716 (2012) 1; Science 338 (2012) 1576.

[2] S. Chatrchyan et al. [CMS Collab.], Phys. Lett. B 716 (2012) 30; Science 338 (2012) 1569.

[3] S. L. Glashow, Nucl. Phys. B22 (1961) 579;

S. Weinberg, Phys. Rev. Lett. 19 (1967) 1264;

A. Salam, in Elementary Particle Theory, ed. N. Svartholm, Amquist and Wiksells, Stockholm (1969), p. 376.

[4] H. Fritzsch, M. Gell-Mann, H. Leutwyler, Phys. Lett. 47 (1973) 365.

H. D. Politzer, Phys. Rev. Lett. 30 (1973) 1346.

D. Gross, F. Wilczek, Phys. Rev. Lett. 30 (1973) 1343.

S. Weinberg, Phys. Rev. Lett. 31 (1973) 494.

[5] P. W. Higgs, Phys. Lett. 12 (1964) 132; Phys. Rev. Lett. 13 (1964) 508;

Phys. Rev. 145 (1966) 1156;

F. Englert, R. Brout, Phys. Rev. Lett. 13 (1964) 321;

G. S. Guralnik, C. R. Hagen, T. W. B. Kibble, Phys. Rev. Lett. 13 (1964) 585 ;

T. W. B. Kibble, Phys. Rev. 155 (1967) 1554.

[6] J. Beringer et al. [Particle Data Group Collab.], Phys. Rev. D 86 (2012) 010001 .

[7] F. Jegerlehner, The Standard model as a low-energy effective theory: what is triggering the Higgs mechanism?, arXiv:1304.7813 [hep-ph].

[8] F. Jegerlehner, The hierarchy problem of the electroweak Standard Model revisited, arXiv:1305.6652 [hep-ph].

[9] P. A. R. Ade et al. [Planck Collab.], arXiv:1303.5062 [astro-ph.CO]; arXiv:1303.5082 [astro-ph.CO].

[10] T. Hambye, K. Riesselmann, Phys. Rev. D 55 (1997) 7255. 
[11] M. Holthausen, K. S. Lim, M. Lindner, JHEP 1202 (2012) 037.

[12] F. Bezrukov, M.Yu. Kalmykov, B.A. Kniehl, M. Shaposhnikov, JHEP 1210 (2012) 140.

[13] G. Degrassi, S. Di Vita, J. Elias-Miro, J. R. Espinosa, G. F. Giudice, G. Isidori, A. Strumia, JHEP 1208 (2012) 098.

[14] S. Alekhin, A. Djouadi, S. Moch, Phys. Lett. B 716 (2012) 214.

[15] L. N. Mihaila, J. Salomon, M. Steinhauser, Phys. Rev. Lett. 108 (2012) 151602 .

[16] K. G. Chetyrkin, M. F. Zoller, JHEP 1206 (2012) 033.

[17] I. Masina, Phys. Rev. D 87 (2013) 5, 053001.

[18] A. V. Bednyakov, A. F. Pikelner, V. N. Velizhanin, JHEP 1301 (2013) 017.

[19] A. V. Bednyakov, A. F. Pikelner, V. N. Velizhanin, Phys. Lett. B 722 (2013) 336.

[20] K. G. Chetyrkin, M. F. Zoller, JHEP 1304 (2013) 091.

[21] A. V. Bednyakov, A. F. Pikelner, V. N. Velizhanin, Nucl. Phys. B 875 (2013) 552.

[22] D. Buttazzo, G. Degrassi, P. P. Giardino, G. F. Giudice, F. Sala, A. Salvio, A. Strumia, JHEP 1312 (2013) 089.

[23] A. V. Bednyakov, A. F. Pikelner, V. N. Velizhanin, Nucl. Phys. B 879 (2014) 256.

[24] F. Jegerlehner, M.Yu. Kalmykov, B.A. Kniehl, Phys. Lett. B 722 (2013) 123.

[25] A. Juste, S. Mantry, A. Mitov, A. Penin, P. Skands, E. Varnes, M. Vos, S. Wimpenny, arXiv:1310.0799 [hep-ph].

[26] M. J. G. Veltman, Acta Phys. Polon. B 12 (1981) 437.

[27] Y. Hamada, H. Kawai, K.Y. Oda, Phys. Rev. D 87 (2013) 053009.

[28] M. S. Al-sarhi, I. Jack, D. R. T. Jones, Z. Phys. C 55 (1992) 283.

[29] D. R. T. Jones, Phys. Rev. D 88 (2013) 098301.

[30] D.A. Kirzhnits, JETP Lett. 15 (1972) 529; D.A. Kirzhnits, A.D. Linde, Phys. Lett. 72B (1972) 471.

[31] S. Weinberg, Phys. Rev. D9 (1974) 3357; L. Dolan, R. Jackiw, Phys. Rev. D9 (1974) 3320; D.A. Kirzhnits, A.D. Linde, JETP 40 (1974) 628. 
[32] D.A. Kirzhnits, A.D. Linde, Ann. Phys. 101 (1976) 195.

[33] M. Dine, R. G. Leigh, P. Y. Huet, A. D. Linde, D. A. Linde, Phys. Rev. D 46 (1992) 550.

[34] Nucl. Phys. B 447 (1995) 317.

[35] K. Kajantie, M. Laine, K. Rummukainen, M. E. Shaposhnikov, Phys. Rev. Lett. 77 (1996) 2887.

[36] V. A. Rubakov, M. E. Shaposhnikov, Usp. Fiz. Nauk 166 (1996) 493 [Phys. Usp. 39 (1996) 461].

[37] D. J. H. Chung, A. J. Long, L. -T. Wang, Phys. Rev. D 87 (2013) 023509.

[38] A. H. Guth, Phys. Rev. D 23 (1981) 347.

[39] A. A. Starobinsky, Phys. Lett. B 91 (1980) 99.

[40] A. D. Linde, Phys. Lett. B 108 (1982) 389, Phys. Lett. B 129 (1983) 177.

[41] A. Albrecht, P. J. Steinhardt, Phys. Rev. Lett. 48 (1982) 1220.

[42] V. F. Mukhanov, G. V. Chibisov, JETP Lett. 33 (1981) 532 [Pisma Zh. Eksp. Teor. Fiz. 33 (1981) 549];

[43] V. F. Mukhanov, JETP Lett. 41 (1985) 493 [Pisma Zh. Eksp. Teor. Fiz. 41 (1985) 402];

[44] V. F. Mukhanov, H. A. Feldman, R. H. Brandenberger, Phys. Rept. 215 (1992) 203.

[45] F. Bezrukov, M. Shaposhnikov, JHEP 0907 (2009) 089.

[46] F. Bezrukov, G. K. Karananas, J. Rubio, M. Shaposhnikov, Phys. Rev. D 87 (2013) 096001.

[47] P. A. R. Ade et al. [Planck Collab.], arXiv:1303.5084 [astro-ph.CO].

[48] A. D. Linde, R. Brandenberger, Inflation and quantum cosmology, Boston, USA: Academic (1990) $199 \mathrm{p}$

[49] E. R. Harrison, Mon. Not. Roy. Astron. Soc. 137 (1967) 69.

[50] E. Galindo-Dellavalle, G. German, A. de la Macorra, arXiv:0804.2240 [grqc].

[51] S. Weinberg, Cosmology, Oxford, UK: Oxford Univ. Pr. (2008) 593 p

[52] E. W. Kolb, M. S. Turner, The Early Universe, Front. Phys. 69 (1990) 1.

[53] N. Cabibbo, Phys. Rev. Lett. 10 (1963) 531.

M. Kobayashi, K. Maskawa, Prog. Theor. Phys. 49 (1973) 652. 
[54] S. Weinberg, Phys. Rev. Lett. 43 (1979) 1566.

[55] W. Buchmüller, D. Wyler, Nucl. Phys. B 268 (1986) 621.

[56] B. Grzadkowski, M. Iskrzynski, M. Misiak, J. Rosiek, JHEP 1010 (2010) 085.

[57] N. Straumann, Eur. J. Phys. 20 (1999) 419.

[58] G. E. Volovik, JETP Lett. 82 (2005) 319 [Pisma Zh. Eksp. Teor. Fiz. 82 (2005) 358].

[59] G. E. Volovik, Int. J. Mod. Phys. D 15 (2006) 1987.

[60] P. A. R. Ade et al. [Planck Collab.], arXiv:1303.5076 [astro-ph.CO];

[61] L.G. Bian, arXiv:1303.2402 [hep-ph].

[62] I. Masina, M. Quiros, Phys. Rev. D 88 (2013) 093003.

[63] Y. Hamada, H. Kawai, K.-y. Oda, Prog. Theor. Exp. Phys. (2014) 023 B02. 